The Astrophysical Journal, 342:735-758, 1989 July 15

(C) 1989. The American Astronomical Society. All rights reserved. Printed in U.S.A.

\title{
A MILLIMETER-WAVE SURVEY OF CO EMISSION IN SEYFERT GALAXIES
}

\author{
T. M. Heckman, ${ }^{1}$ L. Blitz, ${ }^{1}$ A. S. Wilson, AND L. Armus ${ }^{1}$ \\ Astronomy Program, University of Maryland \\ AND \\ G. K. MILEY ${ }^{1}$ \\ Sterrewacht, Leiden \\ Received 1988 July 15 ; accepted 1988 November 9
}

\begin{abstract}
We have used the NRAO $12 \mathrm{~m}$ telescope to search for emission in the $115 \mathrm{GHz} 1-0$ line of CO in a sample of 43 Seyfert galaxies and have detected 18. These galaxies were selected to be bright in the optical, the farinfrared, or both. Together with previously published results, $\mathrm{CO}$ data are now available for 55 Seyfert galaxies (26 detections). The CO properties of an optically selected subset of the Seyfert galaxies, namely those 29 Seyferts in the Revised Shapley Ames Catalog (RSA) having Hubble types from SO/a to Sbc, are compared with the $\mathrm{CO}$ properties of normal galaxies of the same Hubble type. We find that these RSA type 2 Seyferts have an average ratio of CO-to-blue luminosity that is about twice as large as that of the normal galaxies, but the RSA type 1 Seyferts have normal CO luminosities. We also find that the RSA type 2 Seyfert galaxies (but not the RSA type 1 Seyferts) have an unusually large average ratio of CO luminosity-to-H I mass compared to normal disk galaxies (by a factor $\gtrsim 5$ ). The 55 Seyfert galaxies with CO data-as a class-follow the same relationship between $\mathrm{CO}$ and far-IR luminosities as do normal and starburst galaxies, suggesting that the far-IR radiation in this sample of Seyferts has the same origin as in normal and starburst galaxies (dust reradiation of starlight). We have then compared the far-infrared luminosities of all the 42 known Seyfert galaxies in the RSA to those of a sample of non-Seyfert galaxies from the RSA, carefully chosen to match the Seyferts in absolute magnitude, distance, and Hubble type. The RSA type 2 Seyferts have an average farinfrared luminosity that is $\sim 4$ times larger than the non-Seyfert comparison sample, while the RSA type 1 Seyferts are not significantly more luminous than the non-Seyferts. Thus, type 2 Seyferts may have abnormally large dust and molecular gas contents and high rates of star-formation, while the Seyfert 1 galaxies appear normal. This molecular gas may be related in some indirect way to the nuclear material hypothesized to obscure the broad line region in type 2 Seyferts. The differences in CO and far-IR properties also imply that the two classes of Seyfert galaxies are intrinsically different from one another and that the Seyfert 1 population cannot evolve into the Seyfert 2 population in a time scale less than a few million years (or vice versa). We have also searched for any very broad $\mathrm{CO}$ emission lines associated with the high-velocity optical emission-line gas in six Seyfert nuclei. We have been able to put limits on this component that are in some cases a small fraction of the narrower CO emission detected from the galaxy. We find that while Seyfert galaxies exhibit a statistically significant correlation between $\mathrm{CO}$ luminosity and nonthermal radio power, they are systematically stronger nonthermal radio sources than non-Seyfert galaxies having the same CO luminosity. We argue that these results support the idea that the radio continuum in Seyferts has a composite origin with both starburst and active galactic nucleus components. We have also compared the $\mathrm{CO}$ line widths to the widths of the global $\mathrm{H}_{\mathrm{I}} \lambda=21 \mathrm{~cm}$ lines, the nuclear [O III] $\lambda 5007$ lines, and the central stellar velocity dispersions. The CO line widths correlate best with the $\mathrm{H}$ I line widths, and the correlation between the CO and $[\mathrm{O} \mathrm{III}]$ widths improves markedly when the $\mathrm{CO}$ (but not the [O III]) line widths are corrected for galaxy inclination. This suggests that the $\mathrm{CO}$ is coplanar with the galaxy disk (like the $\mathrm{H}$ I), but that the nuclear [O III]-emitting region (the classical narrow line region) is not. The $\mathrm{CO}$ versus [O III] line width correlation provides additional evidence for a gravitational component to the dynamics of the narrow line region.
\end{abstract}

Subject headings: galaxies: interstellar matter - galaxies: Seyfert — radio sources: galaxies

\section{INTRODUCTION}

Two of the most important questions regarding active galactic nuclei (AGNs) concern the mechanism by which the nuclear activity is fueled and the relationship between the activity and the formation of massive stars. This second question is tightly bound up in the controversy concerning the origin of the infrared emission in Seyfert galaxies. In the present paper, we will show how millimeter-wave observations of $\mathrm{CO}$ in a large

\footnotetext{
${ }^{1}$ Visiting observer at the $12 \mathrm{~m}$ telescope of the National Radio Astronomy Observatory, operated by Associated Universities, Inc., under contract with the National Science Foundation.
}

sample of Seyfert galaxies can provide fresh insight into these crucial issues.

The standard picture of AGNs contains two key components: a "central engine" (supermassive black hole) and a "fuel supply" (a source of gas whose gravitational energy can be tapped via accretion). The source of the fuel remains a mystery, but a mounting body of evidence suggests that this source is extrinsic to the nucleus proper-that is, AGNs may be fueled by material brought into the nucleus from the interstellar medium of the host galaxy (see Balick and Heckman 1982). A very promising fuel source is the molecular gas which dominates the ISM in the circumnuclear regions (kiloparsec 
scale) of many spiral galaxies (e.g., Morris and Richard 1982). The specific questions to be answered by observations are then whether Seyfert galaxies have an unusually bountiful supply of circumnuclear molecular gas relative to normal galaxies of similar morphological type and absolute magnitude, and whether the molecular gas is in a dynamically peculiar state. These questions have been considered in two earlier papers dealing with the CO properties of Seyfert galaxies (Bieging et al. 1981; Blitz, Mathieu, and Bally 1986). However, in the present paper, our sample is large enough to address these issues with confidence.

The relationship between star formation and the Seyfert phenomenon has received considerable attention, particularly since data from IRAS have become available. Such a relationship might be expected on a number of grounds. Nuclear activity might trigger star formation as the AGN interacts with the ISM (e.g., Sanders and Bania 1976). Conversely, a burst of star formation might increase the accretion rate of the central engine (Norman 1987) or lead to the formation of the central engine (Weedman 1983; Norman and Scoville 1988). Terlevich and Melnick (1985) have even proposed that a population of young massive stars is the central engine. Even if the star formation and the AGN are not related in a direct causal way, processes that deposit a large amount of molecular gas in the circumnuclear region could well precipitate a starburst and fuel the central engine in parallel. As reviewed recently by Heckman (1988), it has been difficult to prove conclusively that Seyfert galaxies do in fact have a larger than normal rate of star formation. Insofar as molecular gas is the raw material out of which massive stars are formed, it is therefore critical to determine the amount and physical state of molecular gas in Seyferts.

While it has long been known that many Seyfert galaxies are strong infrared sources (see Rieke and Lebofsky 1979), the origin of the infrared is still not well understood. Recent investigations of the IRAS data base have yielded contradictory results. For example, Rodriguez-Espinosa, Rudy, and Jones $(1986,1987)$ favor dust reradiation of light from young stars, while Edelson and Malkan (1986) argue that the infrared is largely nonthermal radiation from the AGN. Miley, Neugebauer, and Soifer (1985), Ward et al. (1987), Edelson, Malkan, and Rieke (1987), Carleton et al. (1987), and Rowan-Robinson (1987) have proposed composite models with young stars and the AGN playing different roles in different Seyferts. Since the far-infrared luminosity and molecular gas content appear to be strongly related in normal and starburst galaxies (e.g., Rickard and Harvey 1984; Sanders et al. 1986), the CO properties of Seyferts may yield valuable clues to the origin of their infrared continuum.

\section{OBSERVATIONS}

\section{a) Sample Selection}

The survey of Young, Scoville, and Brady (1985) indicates that the CO luminosity of spiral galaxies correlates with their blue luminosity, although the relationship shows considerable scatter (Stark et al. 1986). The far-infrared and CO fluxes are also correlated (e.g., Rickard and Harvey 1984). Accordingly, we attempted to observe all the galaxies in Huchra's (unpublished) catalog of Seyferts north of $\delta=-37^{\circ}$, brighter than $B_{T}=13.2$ (in the Revised Shapley Ames Catalog; hereafter RSA, and/or having a flux density greater than $10 \mathrm{Jy}$ at $100 \mu \mathrm{m}$ in the IRAS Point Source Catalog. In total, we observed 29
Seyferts from the RSA, and 20 Seyferts with IRAS flux densities greater than $10 \mathrm{Jy}$ at $100 \mu \mathrm{m}$ (13 Seyferts satisfied both criteria). In some cases, good $\mathrm{CO}$ data had already been published, and these galaxies were only observed if it was convenient. Gaps in the observing schedule were filled by observing seven Seyfert galaxies that were somewhat fainter than the above limits. Forty-three Seyferts were observed in all. The above selection criteria will have important implications concerning the nature of the inferences we can draw concerning the $\mathrm{CO}$ properties of Seyferts. As we will discuss below, we have always attempted to account explicitly for any possible selection effects in our statistical tests.

\section{b) Observations and Data Reduction}

The observations were made at the $12 \mathrm{~m}$ telescope at NRAO located on Kitt Peak over four periods from 1985 May to 1986 October. The data were all obtained by position switching to a reference position separated from the nucleus by $10^{\prime}-15^{\prime}$ in azimuth. All the observations were made with the $256 \times 2$ $\mathrm{MHz}$ filter bank, which gives a velocity coverage of $1330 \mathrm{~km}$ $\mathrm{s}^{-1}$ and a resolution of $5.2 \mathrm{~km} \mathrm{~s}^{-1}$ at $115 \mathrm{GHz}$.

The data from the first three runs were frequently marred by low-level baseline curvature which commonly plagues extragalactic $\mathrm{CO}$ observations. Tests showed that the curvature appeared to result from transients in the system with a characteristic time scale of seconds. Accordingly, we requested that a system which allowed rapid beam switching be installed at the telescope which NRAO kindly accomplished for us prior to our last run in 1986 October. The results were a dramatic improvement in the baselines, to the point that in most sources, no baseline had to be removed from the data. Consequently, the rapid beam switching capability permitted us to determine whether there was any significant contribution to the flux from a broad CO line, wider than the $1330 \mathrm{~km} \mathrm{~s}^{-1}$ instantaneous bandwidth, similar to that seen in the optical line emitting regions of Seyfert nuclei. Such a line would have appeared as a positive offset of the baseline from zero, and was not seen in any of the galaxies.

The weather was generally good for all of the observations, and the SIS receivers produced a single-sideband system temperature of $500-700 \mathrm{~K}$.

Observations taken with the rapid beam switcher required a small upward correction of the observed antenna temperatures because there is no blanking of the signal when the secondary is nutating. This correction amounts to $9 \%$ and is determined by observations of sources small compared to the throw of the secondary ( $6^{\prime}$ maximum) with and without the rapid beam switcher. The values of the line parameters given in the next section include this correction as appropriate. In general, sources which were reobserved from one observing run to the next gave results consistent to within the noise as long as there was no apparent contamination from bad baselines.

The results of these observations are summarized in Table 1 and the associated notes. Spectra of all the detected galaxies are displayed in Figure 1. We detected 18 of the 43 Seyferts, including 11 of the 29 in the RSA, 13 of the 20 with IRAS flux densities greater than $10 \mathrm{Jy}$ at $100 \mu \mathrm{m}$, and three of the seven that satisfied neither the optical nor far-IR selection criteria.

We have then combined our $J=1-0 \mathrm{CO}$ data with data of similar quality in the literature to determine better the general $\mathrm{CO}$ properties of Seyfert galaxies. The assembled data are summarized in Table 2 and are described in the associated notes. 
TABLE 1

OBSERVATIONAL RESULTS

\begin{tabular}{|c|c|c|c|c|c|c|}
\hline $\begin{array}{l}\text { Name } \\
\text { (1) }\end{array}$ & $\begin{array}{c}F_{\mathrm{Co}} \\
(2)\end{array}$ & $\begin{array}{l}V_{\mathrm{Co}} \\
(3)\end{array}$ & $\begin{array}{l}W_{\text {co }} \\
(4)\end{array}$ & $\underset{(5)}{\Delta T_{\text {rms }}}$ & $\begin{array}{l}\text { Observing Runs } \\
\text { (6) }\end{array}$ & $\begin{array}{c}\text { Samples } \\
(7)\end{array}$ \\
\hline Mrk $938=$ NGC $34 \ldots \ldots \ldots \ldots \ldots$ & $5 \pm 2$ & $5810 \pm 75$ & $370 \pm 150$ & 9 & 1,3 & FIR \\
\hline NGC $788 \quad \ldots \ldots \ldots \ldots \ldots \ldots \ldots \ldots \ldots \ldots$ & $<1.5$ & $\ldots$ & & 15 & 4 & RSA \\
\hline NGC $931=$ Mrk $1040 \ldots \ldots \ldots \ldots$ & $1.8 \pm 0.6$ & $4960 \pm 50$ & $500 \pm 100$ & 7 & & \\
\hline NGC $1068 \ldots \ldots \ldots \ldots \ldots \ldots \ldots \ldots$ & $73 \pm 5$ & $1129 \pm 20$ & $310 \pm 20$ & 30 & 3,4 & RSA, FIR \\
\hline NGC $1144 \ldots \ldots \ldots \ldots \ldots \ldots \ldots$ & $<\overline{5}$ & $\ldots$ & $\ldots$ & 9 & 2,3 & FIR \\
\hline Mrk $1066 \ldots \ldots \ldots \ldots$ & $<3$ & $\ldots$ & $\ldots$ & 24 & 1 & FIR \\
\hline NGC $1275 \ldots \ldots \ldots \ldots \ldots \ldots \ldots$ & $<5$ & $\ldots$ & $\ldots$ & 26 & 2,4 & RSA \\
\hline 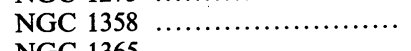 & $<1.8$ & & & 12 & 4 & RSA \\
\hline NGC $1365 \quad \ldots \ldots \ldots \ldots \ldots \ldots \ldots$ & $48 \pm 10$ & $1593 \pm 20$ & $210 \pm 40$ & 44 & 1 & RSA, FIR \\
\hline NGC $1386 \quad \ldots \ldots \ldots \ldots \ldots \ldots \ldots \ldots \ldots$ & $<8$ & & & 27 & 1,4 & RSA \\
\hline NGC $1667 \ldots \ldots$. & $8 \pm 1$ & $4567 \pm 50$ & $460 \pm 100$ & 7 & $1,2,3,4$ & RSA, FIR \\
\hline NGC $2110 \ldots \ldots \ldots$ & $<3$ & & & 12 & 1 & \\
\hline MCG 8-11-11 ..... & $1.3 \pm 0.4$ & $6113 \pm 75$ & $370 \pm 100$ & 6 & 4 & \\
\hline Mrk $3 \ldots \ldots \ldots \ldots \ldots$ & $<1.0$ & & & 6 & 3,4 & \\
\hline NGC $2273=$ Mrk $620 \ldots \ldots \ldots \ldots$ & $2.6 \pm 0.4$ & $1874 \pm 50$ & $280 \pm 100$ & 6 & $1,3,4$ & RSA \\
\hline Mrk $6=$ IC $450 \ldots \ldots \ldots \ldots \ldots \ldots$ & $<5$ & & & 15 & 3 & \\
\hline Mrk $10 \ldots \ldots \ldots \ldots \ldots \ldots \ldots \ldots \ldots$ & $1.8 \pm 0.5$ & $8710 \pm 50$ & $580 \pm 100$ & 8 & 4 & \\
\hline NGC $2639 \ldots \ldots \ldots \ldots$ & $<4$ & $\ldots$ & $\cdots$ & 13 & 3 & RSA \\
\hline NGC $3081 \ldots \ldots \ldots \ldots \ldots \ldots \ldots \ldots$ & $<5$ & $\ldots$ & $\ldots$ & 25 & 3 & RSA \\
\hline NGC $3281 \ldots \ldots \ldots \ldots \ldots \ldots \ldots$ & $<2.4$ & $\ldots$ & $\ldots$ & 19 & 4 & RSA \\
\hline NGC $3516 \ldots \ldots \ldots \ldots \ldots \ldots$ & $<5$ & .. & $\ldots$ & 21 & 1 & RSA \\
\hline NGC $3982 \ldots \ldots \ldots$ & $<5$ & $\ldots$ & $\ldots$ & 9 & 1,3 & RSA, FIR \\
\hline NGC $4235 \ldots \ldots \ldots \ldots \ldots \ldots \ldots$ & $<4$ & $\ldots$ & $\ldots$ & 17 & 3 & RSA \\
\hline NGC $4593 \ldots \ldots \ldots \ldots \ldots \ldots \ldots$ & $<5$ & $\ldots$ & $\ldots$ & 23 & 1 & RSA \\
\hline NGC $4639 \quad \ldots$ & $<5$ & $\ldots$ & $\cdots$ & 22 & 4 & RSA \\
\hline Mrk $231 \ldots \ldots \ldots \ldots \ldots \ldots \ldots \ldots \ldots \ldots \ldots \ldots$ & $3.2 \pm 0.6$ & $12610 \pm 50$ & $415 \pm 100$ & 13 & 4 & FIR \\
\hline 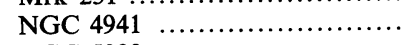 & $<3$ & & & 14 & 3 & RSA \\
\hline NGC $5033 \ldots \ldots \ldots \ldots \ldots \ldots \ldots$ & $11.5 \pm 2$ & $900 \pm 50$ & $460 \pm 100$ & 21 & 1,3 & RSA, FIR \\
\hline NGC $5135 \ldots \ldots \ldots \ldots \ldots \ldots \ldots$ & $10.7 \pm 2$ & $4106 \pm 15$ & $90 \pm 30$ & 17 & 3 & RSA, FIR \\
\hline NGC $5256=$ Mrk $266 \ldots \ldots \ldots \ldots$ & $<5$ & $\ldots$ & $\ldots$ & 16 & 3 & FIR \\
\hline NGC $5273 \ldots \ldots \ldots \ldots \ldots \ldots \ldots \ldots$ & $<5$ & & $\ldots$ & 18 & 3 & RSA \\
\hline 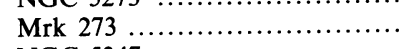 & $2.8 \pm 0.6$ & $11280 \pm 50$ & $450 \pm 150$ & 8 & 3,4 & FIR \\
\hline 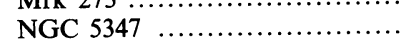 & $2.3 \pm 0.4$ & $2382 \pm 50$ & $140 \pm 100$ & 8 & 4 & RSA \\
\hline NGC $5548 \ldots \ldots \ldots \ldots \ldots \ldots \ldots \ldots \ldots$ & $<10$ & $\ldots$ & $\ldots$ & 23 & 4 & RSA \\
\hline NGC $5728 \ldots \ldots \ldots \ldots \ldots \ldots \ldots \ldots$ & $<3$ & $\ldots$ & $\ldots$ & 18 & 1 & RSA, FIR \\
\hline 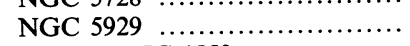 & $<3$ & & $\cdots$ & 12 & 1,4 & RSA, FIR \\
\hline Arp $220=$ IC $4553 \ldots \ldots \ldots \ldots$ & $11.5 \pm 3$ & $5381 \pm 50$ & $480 \pm 100$ & 24 & $\hat{1}$ & FIR \\
\hline NGC $6814 \ldots \ldots \ldots \ldots \ldots \ldots \ldots$ & $2.4 \pm 1$ & $1582 \pm 20$ & $65 \pm 40$ & 15 & 1 & RSA, FIR \\
\hline 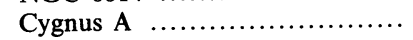 & $<5$ & & & 16 & 3 & \\
\hline IC $5135 \ldots \ldots \ldots$ & $7.6 \pm 0.7$ & $4876 \pm 10$ & $100 \pm 20$ & 16 & 4 & RSA, FIR \\
\hline$\ldots \ldots \ldots \ldots \ldots \ldots \ldots \ldots \ldots$ & $5 \pm 1$ & $2600 \pm 50$ & $600 \pm 100$ & 9 & $2,3,4$ & RSA, FIR \\
\hline NGC $7314 \ldots \ldots \ldots \ldots \ldots \ldots \ldots$ & $<3$ & $\ldots$ & $\ldots$ & 13 & 1,4 & RSA, FIR \\
\hline NGC $7450=$ Mrk $1126 \ldots \ldots \ldots \ldots$ & $<1.8$ & $\ldots$ & $\ldots$ & 13 & 4 & \\
\hline 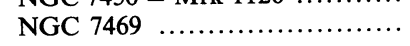 & $12 \pm 1$ & $4927 \pm 40$ & $360 \pm 50$ & 11 & 1,4 & RSA, FIR \\
\hline
\end{tabular}

Key to cols. (2)-(7) follows:
Col. (2).-The CO flux in units of $\mathrm{K} \mathrm{km} \mathrm{s}^{-1}$, on the $T_{R}^{*}$ scale (see $\S \mathrm{II} b$ in the text). Uncertainties are dominated by baseline curvature for runs 1,2 , and 3 (see col. [6]) and are estimated from comparison of the spectra in the left and right filter banks and/or spectra acquired on different days. For run 4 , the uncertainties are produced primarily by channel-to-channel noise and are spectra acquired $N^{1 / 2} \Delta T \quad \Delta v_{\mathrm{CH}}$, where $N$ is the number of channels over which the line is measured, $\Delta T_{\mathrm{rms}}$ is from col. (5), and $\Delta v_{\mathrm{CH}}$ calculated to be $N^{1 / 2} \Delta T_{\text {rms }} \Delta v_{\mathrm{CH}}$, where $N$ is the number of channels over which the line is measured, $\Delta T_{\text {rms }}$, they were empirically is the channel width. For undetected lines, upper limits are taken to be $3 N^{1 / 2} \Delta T_{\text {rms }} \Delta v_{\mathrm{CH}}$ for run 4 , and they were empirically estimated to be 3-5 $\mathrm{K} \mathrm{km} \mathrm{s}^{-1}$ for runs 1,2 , and 3 . When data from

individual values weighted by the inverse square of the uncertainty.
Col. (3).- Heliocentric CO velocity $V_{\mathrm{CO}} \equiv \sum T_{R}^{*}(i) V(i) / \sum T_{R}^{*}(i)$, where the sums are computed over the range in channels in which the line is detected. Uncertainties are estimates only.

Col. (4).-FWHM of the CO emission line in $\mathrm{km} \mathrm{s}^{-1}$. Uncertainties are estimates only.

Col. (5). - The channel-to-channel rms noise (in K) in the sum of all the edited data acquired for each galaxy; $\Delta T_{\mathrm{rms}}$ was computed for channels without $\mathrm{CO}$ line emission.

Col. (6).- The observing runs during which data were obtained. Run 1, 1985 May 30-June 2; Run 2, 1985 Oct 20-22; run 3, 1986 Jan $15-20$; run 4, 1986 Oct 14-18.

Col. (7).- The sample(s) to which the galaxy belongs. Galaxies that are optically bright $\left(B_{T} \leq 13.2\right.$ and therefore in the main catalog or Appendix A of the Revised Shapley-Ames Catalog) are denoted by "RSA." Galaxies that are bright in the far-infrared (IRAS flux density $>10 \mathrm{Jy}$ at $100 \mu \mathrm{m}$ ) are denoted by "FIR." 



FIG. $1 a$

FIG. 1.-Spectra of all the Seyfert galaxies in Table 1 with detected CO emission. The central velocity in $\mathrm{km} \mathrm{s}^{-1}$ (LSR, optical convention) for the filter bank is given below the central fiducial mark in each spectrum. Fiducial marks along the $x$-axis are spaced by $500 \mathrm{~km} \mathrm{~s}^{-1}$ in the galaxy rest frame, except for IC 5135 in which the fiducial marks are spaced by $250 \mathrm{~km} \mathrm{~s}^{-1}$. Velocity (frequency) increases (decreases) to the left in each spectrum. Intensity units along the $y$-axis are the antenna temperatures $(\mathrm{K})$, given in the $T_{R}^{*}$ system. Boxes in each spectrum represent the region of the continuum used to fit and remove a linear baseline. The spectral region over which the CO emission was integrated lies between the boxes, except for Mrk 231 in which the CO line emission is at the extreme left-hand (redshifted) side of the spectrum, and for which only a single continuum box was defined. 

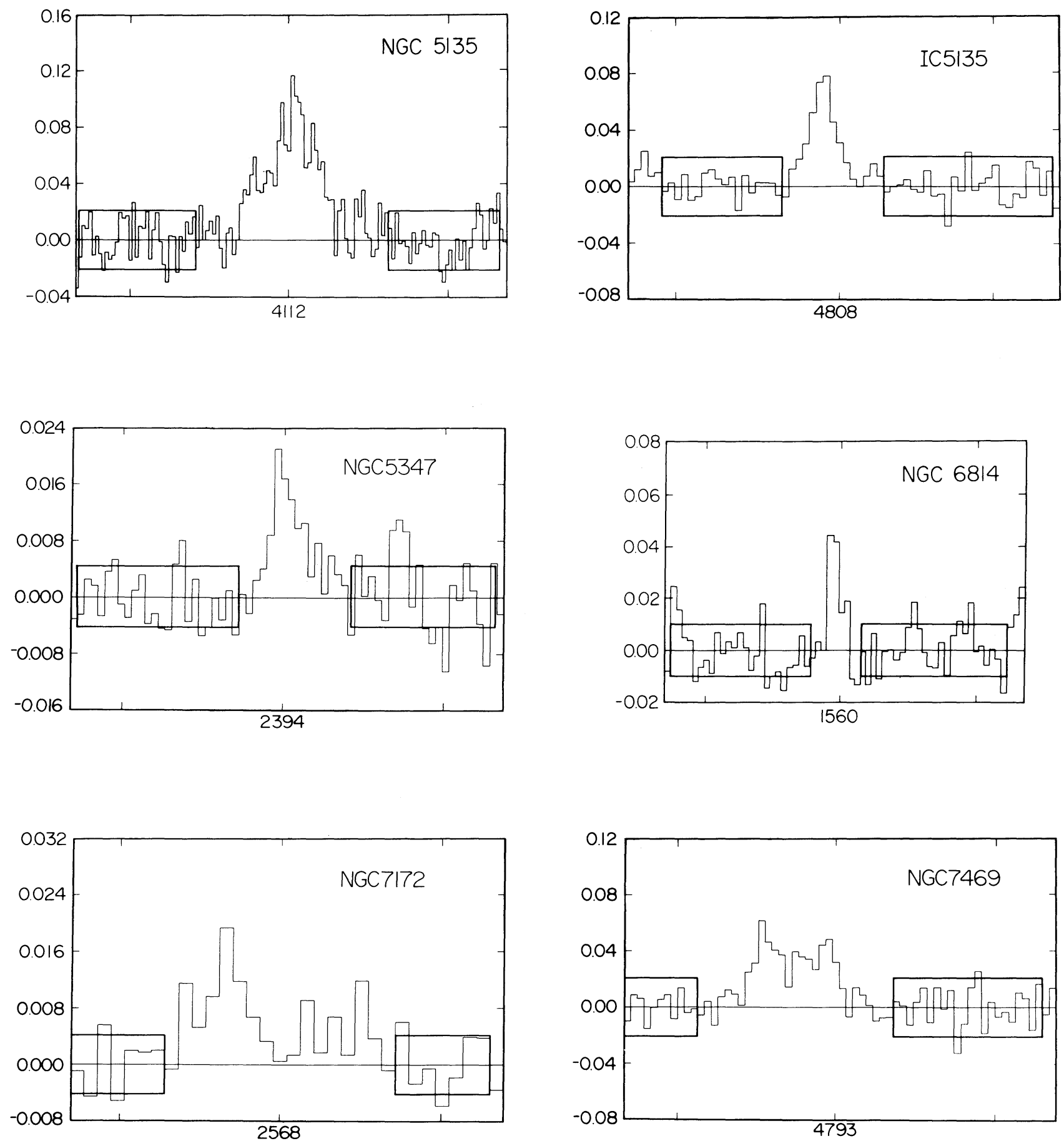

FIG. $1 b$

III. RESULTS

a) The $J=1-0 \mathrm{CO}$ Luminosity

i) Comparison to Normal Galaxies

As emphasized in $\S I$, one of the principal scientific thrusts of this investigation is the comparison of the strength of the $\mathrm{CO}$ emission from Seyfert galaxies with normal galaxies. For such a comparison to be useful, the samples of both Seyfert and normal galaxies must be selected with care.

The sample of Seyfert galaxies best suited to this purpose consists of the 37 known Seyferts in the RSA (either in the main body of the catalog or in Appendix A) with $\mathrm{CO}$ data (18 CO detections), since the surveys of $\mathrm{CO}$ in normal galaxies have also selected galaxies largely on the basis of apparent magnitude. The RSA sample has the important added advantage that good optical magnitudes and morphological types on a uniform system are usually available for the galaxies. We will henceforth designate this as the "RSA CO Seyfert" sample.

In constructing a comparison sample of normal galaxies, we have used several recent surveys whose goal was to determine 


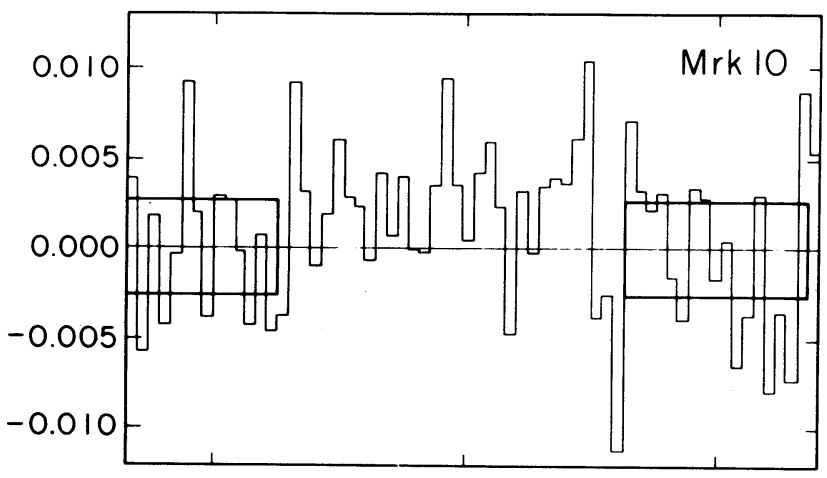

8685
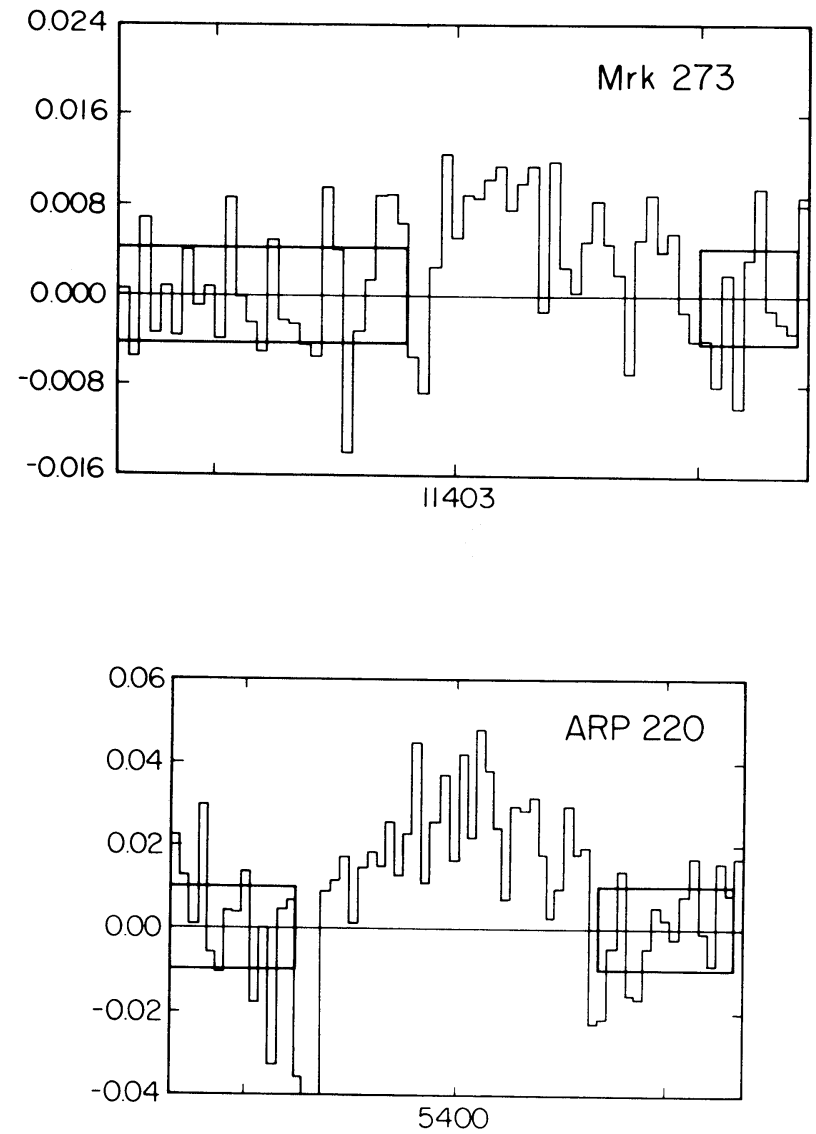

Fig. $1 c$
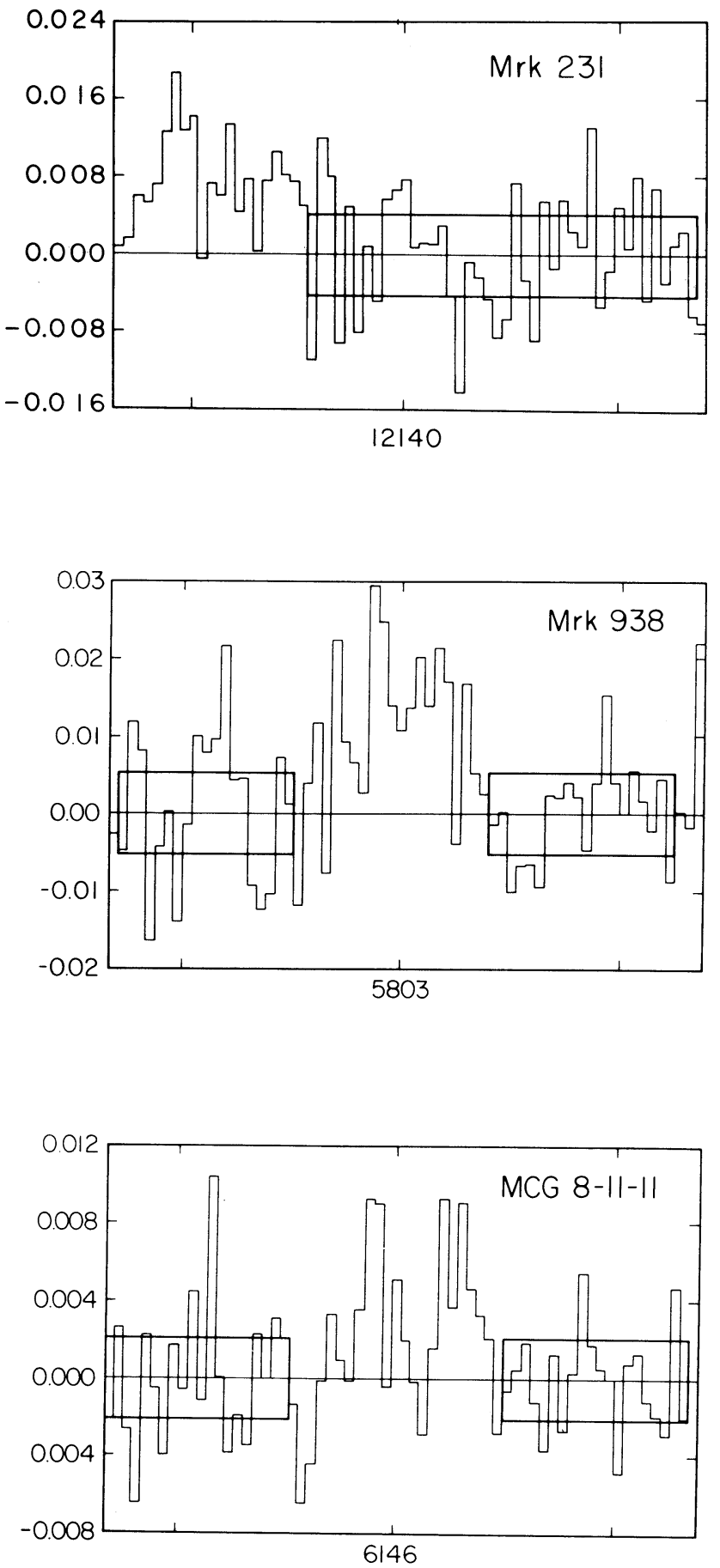

the $\mathrm{CO}$ properties of representative bright galaxies (i.e., galaxies spanning a range of Hubble type and absolute magnitude, but selected on the basis of apparent magnitude). Examples of such surveys are Verter (1985), Young, Scoville, and Brady (1985), Elmegreen and Elmegreen (1982), Stark et al. (1986), and Stark, Elmegreen, and Chance (1987). For both the normal galaxies and the RSA CO Seyferts, the telescope beam size used in the $\mathrm{CO}$ observations is generally smaller than the optical angular extent of the galaxy. Thus, care was taken to ensure that the ratio of the projected beam area to the galaxy optical surface area was the same, on average, for the Seyfert and comparison samples. The optical surface area was taken to be proportional to the blue luminosity of the galaxy (e.g., Holmberg 1975). Unfortunately, this selection procedure excluded the Young, Scoville, and Brady (1985) survey data on Virgo cluster galaxies which were obtained with the FCRAO $14 \mathrm{~m}$ telescope: despite the quality of these data, the projected beam area is much smaller than the average in the RSA CO Seyfert sample. Taking the above sample results in average beam/optical surface areas for the RSA CO Seyfert and non- 
TABLE 2

Summary of CO and Other Relevant Properties of Seyfert Galaxies

\begin{tabular}{|c|c|c|c|c|c|c|c|c|}
\hline$\underset{(1)}{\text { Name }}$ & $\begin{array}{l}c z \\
(2)\end{array}$ & $\begin{array}{l}T_{\text {gal }} \\
(3)\end{array}$ & $\begin{array}{l}T_{\text {Sey }} \\
(4)\end{array}$ & $\begin{array}{l}L_{\mathrm{Co}} \\
(5)\end{array}$ & $\begin{array}{c}M_{B}^{o, i}, i \\
(6)\end{array}$ & $\begin{array}{c}L_{\mathrm{FIR}} \\
(7)\end{array}$ & $\begin{array}{c}P_{1.4} \\
(8)\end{array}$ & $\begin{array}{c}M_{\mathrm{HI}} \\
(9)\end{array}$ \\
\hline Mrk $938=$ NGC 34 & 5803 & pec & 2 & $2.3(1)$ & $(-20.71)$ & 14.3 & & 15 \\
\hline Mrk $348=$ NGC $262 \ldots \ldots \ldots \ldots \ldots$ & 4546 & $\mathrm{Sa}$ & 2 & $<0.64(2)$ & $(-20.21)$ & 0.78 & 1096 & 16 \\
\hline NGC $788^{*} \ldots \ldots \ldots \ldots \ldots$ & 4078 & $\mathrm{Sa} ; \mathrm{SO} / \mathrm{a}$ & 2 & $<0.34(1)$ & -20.76 & 0.22 & 7.4 & \\
\hline NGC $931=$ Mrk $1040 \ldots \ldots \ldots \ldots$ & 4927 & $\mathrm{Sb}$ & 1 & $0.59(1)$ & $(-21.70)$ & 2.1 & $(9.8)$ & 28 \\
\hline NGC $1068 * \ldots \ldots \ldots \ldots \ldots \ldots \ldots \ldots$ & 1153 & $\mathrm{Sb}$ & 2 & $\begin{array}{l}1.3(1) \\
0.7(2) \\
1.4(2)\end{array}$ & -22.05 & 7.4 & 1622 & 3.2 \\
\hline (n. & 8641 & pec & 2 & $<5.2(1)$ & -21.49 & 14.2 & 2516 & \\
\hline Mrk $1066 \ldots \ldots \ldots \ldots \ldots \ldots \ldots \ldots$ & 3611 & so & 2 & $<0.5(1)$ & $(-20.62)$ & 4.0 & 260 & $<0.5$ \\
\hline NGC $1275^{*} \quad \ldots \ldots \ldots \ldots \ldots \ldots \ldots$ & 5268 & Ep; SOp & 2 & $\begin{array}{l}<1.9(1) \\
<3.8(2)\end{array}$ & -22.39 & 4.8 & $1.35 \times 10^{5}$ & $\ldots$ \\
\hline NGC $1358^{*} \ldots \ldots \ldots \ldots \ldots \ldots \ldots$ & 4013 & $\mathrm{Sa} ; \mathrm{SO} / \mathrm{a}$ & 2 & $<0.39(1)$ & -20.75 & 0.28 & 12 & \\
\hline NGC $1365^{*} \ldots \ldots \ldots \ldots \ldots \ldots \ldots$ & 1649 & $\mathrm{Sb}$ & 2 & $1.4(1)$ & -22.07 & 6.8 & 308 & 15 \\
\hline NGC $1386^{*} \ldots \ldots \ldots \ldots \ldots \ldots \ldots \ldots$ & 799 & $\mathrm{Sa}$ & 2 & $<0.2(1)$ & -20.50 & 0.41 & 18 & $<0.4$ \\
\hline NGC $1667^{*}$ & 4578 & Sc & 2 & $2.3(1)$ & -21.66 & 4.9 & 16 & 5.7 \\
\hline NGC $2110 \ldots \ldots \ldots \ldots \ldots \ldots \ldots \ldots$ & 2311 & SO & 2 & $<0.2(1)$ & $(-18.99)$ & 0.77 & 306 & $<0.4$ \\
\hline MCG 8-11-11 $\ldots \ldots \ldots \ldots \ldots \ldots \ldots \ldots$ & 6009 & $\mathrm{Sb}$ & 1 & $0.64(1)$ & $(-22.25)$ & 2.9 & 2399 & 13 \\
\hline Mrk 3 _............................... & 4091 & SO & 2 & $\begin{array}{l}<0.23(1) \\
<2.5(2)\end{array}$ & $(-20.87)$ & 1.6 & 4266 & 4.4 \\
\hline NGC 2273* $=$ Mrk 620 & 1863 & $\mathrm{SO} / \mathrm{a}$ & 2 & $0.12(1)$ & -19.90 & 0.66 & 91 & 1.8 \\
\hline Mrk $6=$ IC $450 \ldots \ldots \ldots \ldots \ldots \ldots$ & 5536 & SO & 1 & $<2.1(1)$ & $(-20.45)$ & 0.92 & 1950 & $<1.4$ \\
\hline 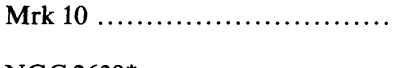 & 8750 & $\mathrm{Sb}$ & 1 & $\begin{aligned} & 1.9(1) \\
< & 10(2)\end{aligned}$ & -22.57 & 2.7 & $<65$ & 38 \\
\hline NGC $2639^{*} .$. & 3226 & Sa & 1 & $\begin{array}{l}<0.6(1) \\
<0.3(10)\end{array}$ & -21.30 & 0.94 & 229 & $\cdots$ \\
\hline NGC 2992* & 2305 & Sa & 2 & $\begin{array}{r}<0.15(2) \\
0.23(3)\end{array}$ & -20.48 & 2.1 & 377 & 5.0 \\
\hline NGC 3081* & 2409 & $\mathrm{Sa} ; \mathrm{SO}$ & 2 & $<0.4(1)$ & -19.79 & $\ldots$ & 3.1 & 4.3 \\
\hline NGC $3227^{*} \ldots \ldots \ldots \ldots \ldots \ldots \ldots$ & 1170 & $\mathrm{Sb} ; \mathrm{Sa}$ & 1 & $\begin{array}{l}0.14(2) \\
0.18(2)\end{array}$ & -19.92 & 0.41 & 32 & 0.9 \\
\hline NGC 3281* & 3393 & SO & 2 & $<0.37(1)$ & -21.23 & 2.0 & 140 & $<5.5$ \\
\hline NGC $3516^{*} \quad \ldots \ldots \ldots \ldots \ldots \ldots \ldots \ldots$ & 2684 & SO & 1 & $<0.5(1)$ & -20.55 & 0.38 & 38 & $<0.6$ \\
\hline NGC $3982 * \ldots \ldots \ldots \ldots \ldots \ldots \ldots$ & 1188 & Sbc; Sc & 2 & $<0.09(1)$ & -19.42 & 0.35 & 35 & 1.3 \\
\hline NGC 4051* $\ldots \ldots \ldots \ldots \ldots \ldots \ldots$ & 713 & Sbc & 1 & $\begin{array}{l}0.029(2) \\
0.059(2)\end{array}$ & -19.43 & 0.20 & 4.4 & 1.4 \\
\hline NGC $4151^{*} \ldots$ & 970 & Sab & 1 & $\begin{array}{l}<0.045(2) \\
<0.012(3)\end{array}$ & -20.05 & 0.16 & 81 & 2.4 \\
\hline NGC $4235^{*}$ & 2300 & $\mathrm{Sa}$ & 1 & $<0.3(1)$ & -20.51 & 0.06 & 11 & $<0.6$ \\
\hline NGC 4388* & 2545 & $\mathrm{Sab} ; \mathrm{Sb}$ & 2 & $\begin{array}{l}0.024(4) \\
0.073(5)\end{array}$ & -20.17 & 0.32 & 46 & 0.4 \\
\hline NGC 4593* & 2698 & $\mathrm{Sb}$ & 1 & $<0.5(1)$ & -21.47 & 0.79 & 7.8 & $<2.6$ \\
\hline NGC 4639* .......... & 970 & $\mathrm{Sb} ; \mathrm{Sbc}$ & 1 & $\begin{array}{l}<0.07(1) \\
<0.01(4) \\
<0.06(5)\end{array}$ & -19.25 & 0.06 & $<4.5$ & 0.85 \\
\hline Mrk $231 \ldots \ldots \ldots \ldots \ldots \ldots \ldots \ldots \ldots \ldots \ldots$ & 12600 & pec & 1 & $\begin{array}{l}6.9(1) \\
2.4(11)\end{array}$ & -22.37 & 128 & 8913 & $<9.3$ \\
\hline NGC 4941* & 720 & Sab & 2 & $<0.02(1)$ & -19.19 & 0.36 & 2.1 & 0.2 \\
\hline NGC 5033* $\ldots \ldots \ldots \ldots$ & 892 & $\mathrm{Sbc} ; \mathrm{Sc}$ & 1 & $\begin{array}{l}0.12(1) \\
0.33(12)\end{array}$ & -20.28 & 0.53 & 34 & 6.5 \\
\hline NGC 5135* & 4112 & $\mathrm{Sb} ; \mathrm{Sab}$ & 2 & $2.4(1)$ & -21.67 & 9.0 & 600 & $<7.9$ \\
\hline M51* ....... & 474 & Sbc & 2 & $\begin{array}{l}0.075(2) \\
0.80(9)\end{array}$ & -20.72 & 0.97 & 98 & 2.0 \\
\hline Mrk $266=$ NGC 5256 & 8239 & pec & 2 & $\begin{array}{r}<4.7(1) \\
2.7(6)\end{array}$ & $(-20.85)$ & 14.8 & 1460 & ... \\
\hline Mrk 273 & 11180 & pec & 2 & $\begin{array}{l}4.9(1) \\
3.0(11)\end{array}$ & -22.04 & 73 & 3459 & $<44$ \\
\hline NGC 5273* & 1089 & $\mathrm{SO} / \mathrm{a} ; \mathrm{SO}$ & 1 & $<0.08(1)$ & -18.35 & 0.04 & $<12.7$ & $<0.03$ \\
\hline NGC 5347* $\ldots \ldots \ldots \ldots$ & 2335 & $\mathrm{Sb} ; \mathrm{Sab}$ & 2 & $0.17(1)$ & -19.66 & 0.28 & 3.7 & $\therefore$ \\
\hline Mrk $463 \ldots$ & 14940 & pec & 2 & $<5.9(2)$ & $(-22.16)$ & 12.8 & $1.8 \times 10^{4}$ & $<2.3$ \\
\hline NGC 5506* & 1753 & $\mathrm{SO} / \mathrm{a}$ & 2 & $\begin{array}{r}<0.10(2) \\
0.13(3)\end{array}$ & -18.67 & 0.70 & 205 & 1.2 \\
\hline NGC $5548^{*} \ldots \ldots \ldots \ldots$ & 5185 & $\mathrm{Sa} ; \mathrm{SO} / \mathrm{a}$ & 1 & $<3.6(1)$ & -21.20 & 0.86 & 150 & 0.9 \\
\hline NGC 5728* & 2834 & $\mathrm{Sb} ; \mathrm{Sa}$ & 2 & $<0.3(1)$ & -21.59 & 2.24 & 120 & 3.6 \\
\hline NGC 5929* & 2504 & Sabp & 2 & $<0.3(1)$ & -20.42 & 1.7 & 258 & 0.8 \\
\hline $\operatorname{Arp} 220=$ IC $4553 \ldots$. & 5400 & pec & 2 & $\begin{array}{l}4.6(1) \\
2.3(3) \\
2.4(7)\end{array}$ & $(-20.16)$ & 84 & 2623 & $<1.0$ \\
\hline NGC 6814* $\ldots \ldots \ldots \ldots \ldots \ldots \ldots \ldots \ldots \ldots \ldots \ldots$ & 1552 & Sbc & 1 & $\begin{array}{l}0.077(1) \\
0.087(2) \\
0.22(2) \\
0.17(3)\end{array}$ & -20.33 & 0.66 & 89 & 3.3 \\
\hline
\end{tabular}


TABLE 2-Continued

\begin{tabular}{|c|c|c|c|c|c|c|c|c|}
\hline $\begin{array}{l}\text { Name } \\
\text { (1) }\end{array}$ & $\begin{array}{l}c z \\
\text { (2) }\end{array}$ & $\begin{array}{l}T_{\text {gal }} \\
\text { (3) }\end{array}$ & $\begin{array}{l}T_{\text {Sey }} \\
\text { (4) }\end{array}$ & $\begin{array}{c}L_{\mathrm{Co}} \\
(5)\end{array}$ & $\begin{array}{c}M_{B_{T}}^{o, i} \\
(6)\end{array}$ & $\begin{array}{l}L_{\mathrm{FIR}} \\
(7)\end{array}$ & $\begin{array}{l}P_{1.4} \\
(8)\end{array}$ & $\begin{array}{c}M_{\mathrm{HI}} \\
(9)\end{array}$ \\
\hline Cygnus A & 17080 & $\mathrm{cDp}$ & 2 & $<21(1)$ & -21.62 & $\lesssim 18$ & $9.8 \times 10^{7}$ & \\
\hline IC $5135^{*} \ldots$ & 4796 & $\mathrm{Sa} ; \mathrm{pec}$ & 2 & $2.4(1)$ & -21.39 & 11.9 & $\ldots$ & $<10$ \\
\hline NGC 7172* & 2568 & $\mathrm{Sab}$ & 2 & $0.44(1)$ & -20.38 & 1.2 & 28 & $<0.6$ \\
\hline NGC 7314* $\ldots \ldots \ldots \ldots$ & 1626 & $\mathrm{Sc} ; \mathrm{Sbc}$ & 1 & $<0.1(1)$ & -20.38 & 0.58 & 20 & 4.2 \\
\hline NGC $7450=$ Mrk $1126 \ldots \ldots \ldots \ldots$ & 3103 & SOp & 2 & $<0.23(1)$ & $(-18.84)$ & & 8.1 & 3.6 \\
\hline NGC 7469* $\ldots \ldots \ldots \ldots \ldots \ldots \ldots$ & 4793 & $\mathrm{Sab} ; \mathrm{Sa}$ & 1 & $\begin{array}{l}3.7(1) \\
1.5(3)\end{array}$ & -22.22 & 18.2 & 1072 & 2.6 \\
\hline NGC $7674^{*}=$ Mrk $533 \ldots$ & 8698 & & & $1.1(8)$ & & & & \\
\hline NGC $7682 \ldots \ldots \ldots \ldots \ldots$ & $\begin{array}{l}8098 \\
5100\end{array}$ & $\begin{array}{l}\text { Sbc } \\
\text { Sab }\end{array}$ & $\begin{array}{l}2 \\
2\end{array}$ & $\begin{array}{c}2.3(3) \\
<0.17(3)\end{array}$ & $\begin{array}{l}-22.49 \\
-2006\end{array}$ & 13.6 & 2630 & 24 \\
\hline
\end{tabular}

Key to cols. (1)-(9) follows:

Col. (1).-Common name(s). Asterisk denotes membership in the Revised Shapley-Ames Catalog of Bright Galaxies (RSA).

Col. (2).- Heliocentric redshift, from Huchra (private communication), the RSA, or the Second Reference Catalogue of Bright Galaxies (de Vaucouleurs, de Vacouleurs, and Corwin 1976; hereafter RC2.

Col. (3).- Galaxy morphological type taken from the RSA or RC2 when available. When two types are given, the RSA and RC2 disagree (RSA type given first). When no RSA or RC2 type is available, the type is taken from the Uppsala General Catalogue of Galaxies (Nilson 1971; hereafter UGC), or estimated by us using the Palomar Observatory Sky Survey or our unpublished direct images.

Col. (4).- Seyfert type, taken from Huchra (private communication).

Col. (5).- The CO luminosity in units of thousands of $\mathrm{K} \mathrm{km} \mathrm{s}^{-1} \mathrm{kpc}^{2} . L_{\mathrm{co}}$ is defined to be the product of $F_{\mathrm{co}}$ (see key to references below) and the projected telescope beam area (in $\mathrm{kpc}^{2}$ ) for the galaxy observed. The angular beam area at $115 \mathrm{GHz}$ is taken to be $1000 \pi$ arcsec ${ }^{2}$ for the new NRAO $12 \mathrm{~m}$ telescope $1089 \pi \operatorname{arcsec}^{2}$ for the old NRAO $12 \mathrm{~m}$ telescope, $506 \pi \operatorname{arcsec}^{2}$ for the University of Massachusetts FCRAO telescope, and $2500 \pi \operatorname{arcsec}^{2}$ for the $7 \mathrm{~m}$ Bell Laboratories telescope. The conversion from angular to physical beam area was computed using the distances implied by the heliocentric redshift in Col. (2) and an assumed Hubble constant of $H_{0}=75 \mathrm{~km} \mathrm{~s} \mathrm{~s}^{-1} \mathrm{Mpc}$. The exceptions are NGC 1365 and NGC 1386 (in Fornax cluster, assumed distances = $20 \mathrm{Mpc}$ ), and NGC 4388 and NGC 4639 (in Virgo cluster, assumed distance $=14 \mathrm{Mpc}$ )

Key to the references used for the CO fluxes is as follows: (1) This paper. (2) Blitz, Mathieu, and Bally 1986. (3) Sanders and Mirabel 1985. (4) Young, Scoville, and Brady 1985. (5) Stark et al. 1986. (6) Sanders et al. 1986. (7) Young et al. 1984. (8) Young et al. 1986. (9) Scoville and Young 1983. (10) Verter 1985.(11) Sanders et al. 1988. (12) Stark, Elmegreen, and Chance 1987.

Col. (6).-Absolute blue magnitude $\left(H_{0}=75\right)$, corrected for Galactic and internal extinction as described in the RSA. When an RSA $B_{T}^{0, i}$ magnitude was not available, an equivalent RC2 $B_{T}^{0}$ magnitude was used. For 12 galaxies, no magnitude on the $B_{T}$ scale was available. For these we have used the Zwicky magnitudes, have placed them on the $B_{T}$ scale following Fisher and Tully 1981 , and have then corrected them for internal and Galactic extinction following the precepts of the RSA. These values for $M_{B_{T}}^{0, i}$ are given in parentheses and are quite uncertain $( \pm 0.5 \mathrm{mag})$.

Col. (7).-Far-infrared luminosity in units of $10^{10} L_{\odot}$ computed using the far-infrared flux (as defined in Lonsdale et al. 1985) and the distance discussed in the notes to col. (5).

Col. (8).- Nonthermal radio continuum power at $v=1.4 \mathrm{GHz}$ in units of $10^{20} \mathrm{~W} \mathrm{~Hz} \mathbf{H z}^{-1}$. We have taken radio fluxes measured with the largest available radio beam to insure maximum sensitivity to large-scale (galaxy-scale) radio structure (e.g., single-dish measurements, VLA C- or D-configuration, Westerbork etc.). Radio powers in parentheses are from VLA A-configuration data and hence may underestimate the total radio power of the galaxy. References for the radio data are Condon 1988, Condon 1980, Condon and Broderick 1988, Dressel and Condon 1978, Condon et al. 1982, de Vaucouleurs, de Vaucouleurs, and Corwin 1976, Edelson 1987, Hummel 1980, Kellermann, PaulinyToth, and Williams 1969, Kojian et al. 1976, Meurs and Wilson 1984, Sulentic 1976, Tovmassian 1966, Ulvestad and Wilson 1984, Wilson and Meurs 1982, Wilson and Ulvestad 1982, and Wilson (unpublished).

Col. (9).- The $\mathrm{H}$ I mass in units of $10^{9} M_{\odot}$ taken from Huchtmeier et al. 1983 and references therein, Heckman, Balick, and Sullivan 1978, Mirabel (private communication), or Mirabel and Wilson 1984, adjusted to our choice of $H_{0}=75 \mathrm{~km} \mathrm{~s}^{-1} \mathrm{Mpc}^{-1}$.

Seyfert samples that agree to within a factor of $\sim 1.5$ for the two samples (with the slightly larger ratio pertaining to the comparison galaxy sample). The relevant properties of the comparison sample of normal galaxies are listed in Table 3.

We have compared the ratio of the CO-to-blue luminosity as a function of Hubble type for the RSA CO Seyfert and normal galaxy samples (Fig. 2). We have also displayed the same information using all the 55 Seyferts in Table 2 . As is evident in the figure, there is a significant overlap in Hubble type between the RSA CO Seyfert and comparison samples only over the relatively narrow range in type from $\mathrm{SO} / \mathrm{a}$ to $\mathrm{Sbc}$ (few Seyferts are of later type, and few data on $\mathrm{CO}$ emission from very early-type normal galaxies exist). Summing over this range (Fig. 3), the mean value of $L_{\mathrm{CO}} / L_{B}$ is larger by a factor of $\sim 1.5$ for the RSA CO Seyfert galaxies, a difference which is significant at the $98 \%$ confidence level (see Table 4 for details). Since the average ratio of telescope beam-to-galaxy surface area was 1.5 times larger for the comparison sample, the true excess in $L_{C O} / L_{B}$ for the RSA CO Seyferts may be somewhat larger than the above (perhaps by a factor 1.5).
The comparison changes in a dramatic way when we compare the type 1 and type 2 RSA CO Seyferts separately. As illustrated in Figure 4 and summarized in Table 4, the type 1 RSA CO Seyferts considered alone do not have a significant excess in $L_{\mathrm{CO}} / L_{B}$, compared to the normal galaxies. In contrast, the type 2 RSA CO Seyfert galaxies have an average $L_{\mathrm{CO}} / L_{B}$ that is higher than the corresponding ratio for normal galaxies by a factor of 2.1 (difference significant at the $99.5 \%$ confidence level). This effect does not reflect any significant difference in the beam sizes for the type 1 and type 2 Seyferts.

In some type 1 Seyfert galaxies, nonthermal radiation from the nucleus makes a significant contribution to the total blue luminosity of the galaxy. However, the systematically small ratio of $L_{\mathrm{CO}} / L_{B}$ for the type 1 RSA CO Seyferts (compared to the type 2 RSA CO Seyferts) is caused by a low $L_{\mathrm{CO}}$ rather than a nucleus-enhanced $L_{B}$. This is clear from two lines of evidence. First, the typical nuclear contribution to $L_{B}$ is only of order $\lesssim 10 \%$ for RSA type 1 Seyferts (Malkan and Filippenko 1983), much less than the factor of 2 difference in $L_{C O} / L_{B}$. Second, the systematic difference between the type 1 and type 2 Seyferts is 
TABLE 3

CO Properties of Comparison SAMple

\begin{tabular}{|c|c|c|c|c|c|c|}
\hline $\begin{array}{c}\text { Name } \\
\text { (1) }\end{array}$ & $\begin{array}{l}c z \\
(2)\end{array}$ & $\begin{array}{l}T_{\mathrm{gal}} \\
(3)\end{array}$ & $\begin{array}{l}L_{\mathrm{CO}} \\
\text { (4) }\end{array}$ & $\begin{array}{c}M_{B_{T}}^{0, i} \\
(5)\end{array}$ & $\begin{array}{c}\log \left(L_{\mathrm{CO}} / L_{B}\right) \\
(6)\end{array}$ & $\begin{array}{l}\text { Reference } \\
\text { (7) }\end{array}$ \\
\hline NGC 613 . & 1534 & $\mathrm{Sb} ; \mathrm{Sbc}$ & 0.62 & -21.36 & -7.96 & 1 \\
\hline NGC 1097. & 1284 & $\mathrm{Sbc} ; \mathrm{Sb}$ & 0.21 & -21.42 & -8.41 & 1 \\
\hline NGC $1300 \ldots \ldots \ldots \ldots$ & 1526 & $\mathrm{Sb} ; \mathrm{Sbc}$ & $<0.16$ & -21.11 & $<-8.43$ & 1 \\
\hline NGC $1425 \ldots \ldots \ldots \ldots$ & 1440 & $\mathrm{Sb}$ & 0.48 & -20.63 & -7.76 & 2 \\
\hline NGC 2782 . & 2574 & $\mathrm{Sa}$ & 0.47 & -21.16 & -7.98 & 2 \\
\hline NGC 3147 . & 2899 & $\mathrm{Sb} ; \mathrm{Sbc}$ & 1.0 & -22.04 & -8.00 & 2 \\
\hline NGC $3169 \ldots$. & 1067 & $\mathrm{Sb} ; \mathrm{Sa}$ & 0.17 & -20.19 & -8.04 & 2 \\
\hline NGC $3310 \ldots$ & 1073 & $\mathrm{Sbc}$ & $<0.062$ & -19.94 & $<-8.37$ & 1 \\
\hline NGC 3344 . & 627 & Sbc & 0.021 & -19.42 & -8.64 & 1 \\
\hline NGC 3351. & 641 & $\mathrm{Sb}$ & $<0.028$ & -19.78 & $<-8.66$ & 1 \\
\hline NGC 3642 . & 1733 & $\mathrm{Sb} ; \mathrm{Sbc}$ & 0.045 & -20.79 & -8.73 & 2,3 \\
\hline NGC 3675 . & 792 & $\mathrm{Sb}$ & $<0.051$ & -19.98 & $<-8.48$ & 2 \\
\hline NGC 3718 . & 1082 & $\mathrm{Sa}$ & $<0.081$ & -20.36 & $<-8.42$ & 1 \\
\hline NGC 3898 . & 1258 & $\mathrm{Sa} ; \mathrm{Sab}$ & $<0.079$ & -20.15 & $<-8.35$ & 2 \\
\hline NGC $3976 .$. & 2333 & $\mathrm{Sc} ; \mathrm{Sb}$ & $<0.31$ & -20.89 & $<-8.07$ & 4 \\
\hline NGC 3992 . & 1134 & $\mathrm{Sb} ; \mathrm{Sbc}$ & $<0.089$ & -20.95 & $<-8.62$ & 1 \\
\hline NGC 4158 . & 2377 & $\mathrm{Sa} ; \mathrm{Sb}$ & 0.21 & -20.16 & -7.92 & 4 \\
\hline NGC $4192 .$. & V & $\mathrm{Sb} ; \mathrm{Sab}$ & 0.15 & -20.88 & -8.37 & 4 \\
\hline NGC $4206 .$. & V & Sbc & $<0.045$ & -18.13 & $<-7.80$ & 4 \\
\hline NGC $4216 \ldots$ & V & $\mathrm{Sb}$ & $<0.064$ & -20.97 & $<-8.77$ & 4 \\
\hline NGC $4314 .$. & 850 & $\mathrm{Sa}$ & $<0.050$ & -18.92 & $<-8.06$ & 1 \\
\hline NGC $4343 .$. & V & $\mathrm{Sb}$ & $<0.032$ & -17.64 & $<-7.74$ & 4 \\
\hline NGC $4351 \ldots$ & V & $\mathrm{Sab}$ & $<0.023$ & -18.26 & $<-8.60$ & 4 \\
\hline NGC $4394 .$. & v & $\mathrm{Sb}$ & $<0.036$ & -19.45 & $<-8.41$ & 4 \\
\hline NGC 4402 . & V & $\mathrm{Sb}$ & 0.17 & -18.43 & -7.33 & 4 \\
\hline NGC $4413 .$. & V & $\mathrm{Sb}$ & $<0.033$ & -17.40 & $<-7.67$ & 4 \\
\hline NGC $4438 \ldots$ & V & $\mathrm{Sb} ; \mathrm{SO} / \mathrm{a}$ & 0.12 & -20.76 & -8.41 & 4 \\
\hline NGC 4440 . & V & $\mathrm{Sa}$ & $<0.061$ & -18.17 & $<-7.66$ & 4 \\
\hline NGC 4450 . & $\mathrm{V}$ & Sab & $<0.027$ & -20.41 & $<-8.82$ & 2,4 \\
\hline NGC 4452 . & V & $\mathrm{SO} ; \mathrm{Sa}$ & $<0.061$ & -17.43 & $<-7.37$ & 4 \\
\hline NGC $4501 .$. & V & $\mathrm{Sbc} ; \mathrm{Sb}$ & 0.46 & -20.94 & -7.91 & 2,4 \\
\hline NGC 4527 .. & V & $\mathrm{Sb} ; \mathrm{Sbc}$ & 0.64 & -20.43 & -7.55 & 4 \\
\hline NGC $4548 \ldots \ldots \ldots \ldots$ & V & $\mathrm{Sb}$ & 0.078 & -20.30 & -8.43 & 4 \\
\hline NGC $4569 \ldots \ldots \ldots \ldots$ & V & $\mathrm{Sab}$ & 0.43 & -21.34 & -8.10 & 4 \\
\hline NGC $4579 \ldots \ldots \ldots \ldots$ & V & $\mathrm{Sab} ; \mathrm{Sb}$ & 0.10 & -20.72 & -8.48 & 4 \\
\hline NGC $4586 \ldots \ldots \ldots \ldots$ & V & $\mathrm{Sa}$ & $<0.073$ & -19.20 & $<-8.01$ & 4 \\
\hline NGC $4694 \ldots \ldots \ldots \ldots$ & V & SO & $<0.042$ & -18.52 & $<-8.00$ & 4 \\
\hline NGC $4698 \ldots \ldots \ldots \ldots . . . . .$. & V & $\mathrm{Sa} ; \mathrm{Sab}$ & $<0.070$ & -19.58 & $<-8.18$ & 2,4 \\
\hline NGC $5383 \ldots \ldots \ldots \ldots$ & 2322 & $\mathrm{Sb}$ & $<0.36$ & -20.91 & $<-8.00$ & $\overrightarrow{1}$ \\
\hline NGC $5701 \ldots \ldots \ldots \ldots$ & 1424 & $\mathrm{Sa} ; \mathrm{SO} / \mathrm{a}$ & $<0.045$ & -19.59 & $<-8.37$ & 3 \\
\hline NGC $5905 \ldots \ldots \ldots \ldots$ & 3544 & $\mathrm{Sb}$ & $<0.16$ & -21.60 & $<-8.62$ & 3 \\
\hline NGC $6951 \ldots \ldots \ldots \ldots$. & 1710 & $\mathrm{Sb} ; \mathrm{Sbc}$ & 0.24 & -20.48 & -8.00 & 1 \\
\hline NGC $7217 \ldots \ldots \ldots \ldots$ & 1234 & $\mathrm{Sb} ; \mathrm{Sab}$ & 0.13 & -20.78 & -8.37 & 3 \\
\hline NGC $7371 \ldots \ldots \ldots \ldots$ & 2826 & $\mathrm{Sa} ; \mathrm{SO} / \mathrm{a}$ & 2.5 & -20.30 & -6.91 & 3 \\
\hline NGC $7479 \ldots \ldots \ldots \ldots$ & 2650 & $\mathrm{Sbc}$ & $<0.47$ & -21.47 & $<-8.10$ & 1 \\
\hline NGC $7716 \ldots \ldots \ldots \ldots$ & 2735 & $\mathrm{Sab} ; \mathrm{Sb}$ & $<0.35$ & -20.38 & $<-7.80$ & 3 \\
\hline NGC $7742 \ldots \ldots \ldots \ldots$ & 1901 & $\mathrm{Sa} ; \mathrm{Sb}$ & 0.18 & -20.03 & -7.95 & 3 \\
\hline
\end{tabular}

Key to cols. (1)-(7) follows:

Cols. (1)-(5).-See notes to Table 2. "V" in col. (2) means membership in the Virgo cluster was assumed (distance $=14 \mathrm{Mpc}$ ).

Col. (6).-The log of the ratio of the CO luminosity $\left(\mathrm{K} \mathrm{km} \mathrm{s}^{-1} \mathrm{kpc}^{2}\right)$ to blue luminosity $\left(L_{\odot}\right)$.

Col. (7).-References for CO data. (1) Elmegreen and Elmegreen 1982. (2) Stark, Elmegreen, and Chance 1987. (3) Verter 1985. (4) Stark et al. 1986.

still present when $L_{\mathrm{Co}}$ is normalized by the galaxy $\mathrm{H}$ I mass rather than by $L_{B}$ (as we will now discuss).

The other major constituent of the interstellar medium in the disks of galaxies is the $\mathrm{H}$ I. Previous investigations have shown no evidence for a systematic surplus or deficit of $\mathrm{H}_{\mathrm{I}}$ in Seyferts relative to normal galaxies (Heckman, Balick, and Sullivan 1978; Mirabel and Wilson 1984), contrary to the above results for the CO. Thus, it is important to assess the relationship between the molecular and atomic hydrogen in Seyfert galaxies and to compare this relationship to that for normal galaxies.

For the same set of normal galaxies which were compared to the RSA CO Seyferts in terms of the CO-to-blue luminosity ratio (see above), we have assembled data on the $\mathrm{H}$ I masses (Huchtmeier et al. 1983, and references therein). We have excluded from this comparison sample the galaxies in Stark et al. (1986) that are found within a projected radius of $\sim 1 \mathrm{Mpc}$ (4.5) from the center of the Virgo cluster because Stark et al. found them to be deficient in $\mathrm{H} \mathrm{I}$ (presumably due to stripping by the intracluster medium). We have then calculated the log of the ratio of the CO luminosity $\left(\mathrm{K} \mathrm{km} \mathrm{s}^{-1} \mathrm{kpc}^{2}\right)$ to $\mathrm{H}$ I mass (solar units) for the RSA CO Seyfert and normal galaxies.

The results are summarized in Figure 5 and Table 4 . In agreement with the results above, it is the type 2 RSA CO 


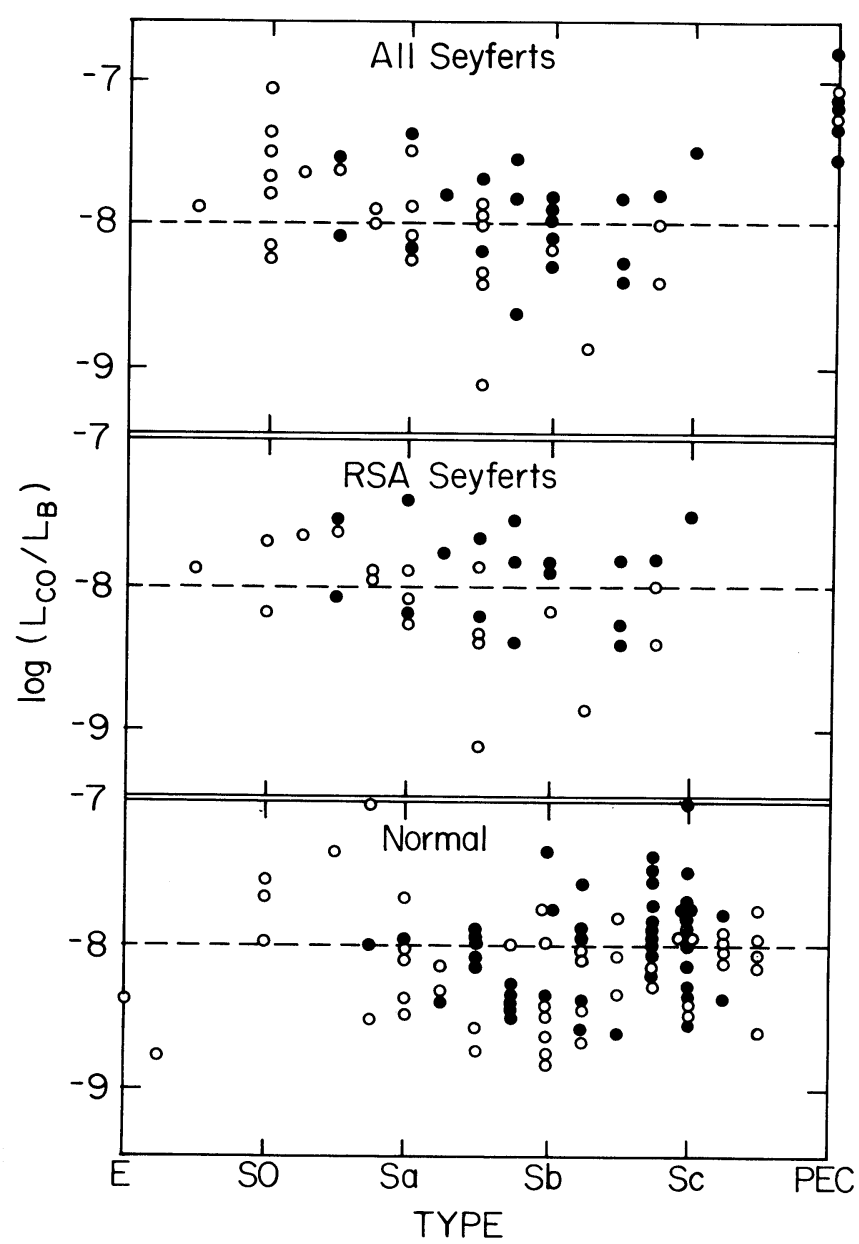

FIG. 2.-The $\log$ ratio of the $\mathrm{CO}$ luminosity (in $\mathrm{K} \mathrm{km} \mathrm{s}^{-1} \mathrm{kpc}^{2}$ ) to the blue luminosity (solar units) plotted as a function of Hubble type for all the Seyfert galaxies in Table 2 (top), only those Seyfert galaxies in the Revised ShapleyAmes Catalog (middle), and normal galaxies (bottom) from Verter (1985), Stark et al. (1986), Elmegreen and Elmegreen (1982), and Stark, Elmegreen, and Chance (1987). CO detections are denoted by solid dots, while upper limits (CO nondetections) are denoted by hollow dots. The normal galaxies extend in Hubble type to Scd (see the text for details concerning the sample), while the top Seyfert sample includes seven galaxies too peculiar in optical morphology to classify on the Hubble sequence.

Seyferts that are unusual: relative to the normal spirals, the average ratio of $L(\mathrm{CO}) / M(\mathrm{H} \mathrm{I})$ is $\sim 5$ times larger for the type 2 Seyferts. In contrast, the average $L(\mathrm{CO}) / M(\mathrm{H}$ I) for the type 1 RSA CO Seyferts does not differ significantly from that for normal galaxies. This result is then quite compatible with the earlier H I surveys of Seyfert galaxies since it suggests that the type 1 Seyferts are normal in terms of both their atomic and molecular gas, while the type 2 Seyferts are only unusual in the properties of their molecular gas.

We note that the standard conversion factor between $L(\mathrm{CO})$ and molecular hydrogen mass (e.g., Bloemen et al. 1986; Israel and Rowan-Robinson 1984) implies that $M(\mathrm{H} \mathrm{I})=M\left(\mathrm{H}_{2}\right)$ for $\log [L(\mathrm{CO}) / M(\mathrm{H} \mathrm{I})]=-6.8$ in our units. About $38 \%$ (nine of 24) of all (RSA or otherwise) the type 2 Seyferts would then have more measured molecular gas than atomic gas, compared to only $10 \%$ (four of 42 ) of the type 1 Seyferts and normal galaxies. It is not clear, however, that the use of this standard conversion factor is warranted, as we will discuss below. ii) Relationship to the Far-Infrared Luminosity

Several recent papers have stressed the strong relation between the strength of the $\mathrm{CO}$ and far-infrared (FIR) continuum in normal and infrared-bright galaxies (e.g., Rickard and Harvey 1984; Sanders et al. 1986; Young et al. 1986). This correlation is usually interpreted as the result of the link between the amount of molecular gas (potential star-forming material) and the rate of star formation. Two of our main goals were to better understand both the origin of the FIR and the relationship between star formation and Seyfert activity. Clearly, the first step is to determine whether Seyfert galaxies obey the same FIR/CO relation as other galaxies.

Sanders et al. (1986) find that the ratio of the CO and FIR luminosities is a function of the FIR luminosity, decreasing at large $L_{\mathrm{FIR}}$. Accordingly, in Figure 6 we have plotted $L_{\mathrm{CO}} / L_{\mathrm{FIR}}$ versus $L_{\mathrm{FIR}}$ for all the Seyferts in Table 2, for the normal galaxies in Stark et al. (1986) and Stark, Elmegreen, and Chance (1987), and for the FIR-bright non-Seyfert from Sanders and Mirabel (1985), Sanders et al. (1986), and Young et al. (1984, 1986). There is no evidence that the Seyfert galaxies depart systematically from the other galaxies in Figure 6. Over the range of $\log \left(L_{\mathrm{FIR}}\right)=9.5-11.5$, where there is good overlap between the Seyfert and non-Seyfert samples, the average value of $\log \left(L_{\mathrm{CO}} / L_{\mathrm{FIR}}\right)$ is only 0.08 larger for the Seyferts, a difference significant at only the $60 \%$ confidence level (see Table 4). In particular, if the FIR continuum in most of the Seyfert galaxies in our sample were largely powered by the AGN (nonthermal

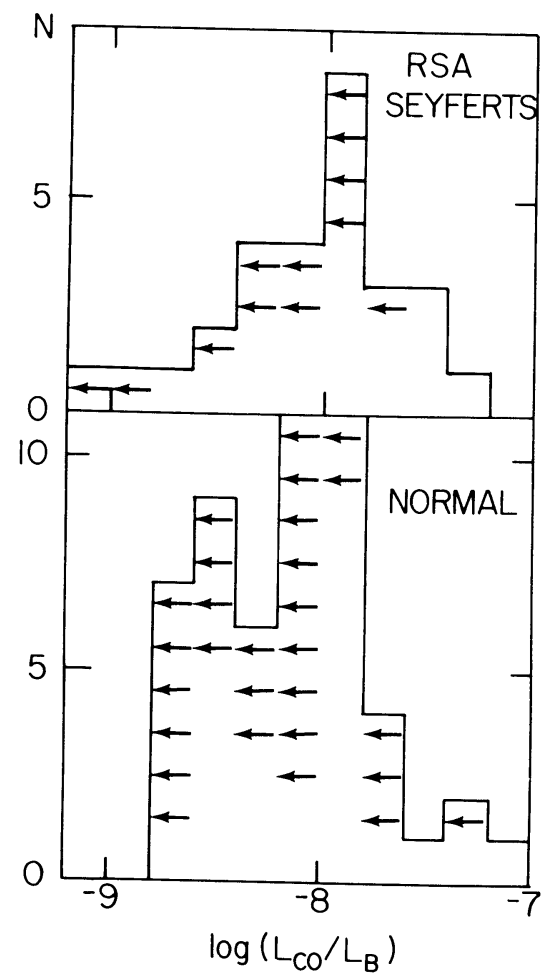

Fig. 3.-Histograms of the $\log$ of the CO-to-blue luminosity ratio for Seyfert galaxies in the Revised Shapley-Ames Catalog (top) and normal galaxies (bottom). All galaxies plotted have Hubble types from SO/a to Sbc (see the text and Tables 2 and 3 for details). The RSA Seyferts have an average $L_{\mathrm{co}} / L_{B}$ that is 1.5 times larger than the normal galaxies (see Table 4). 
TABLE 4

SUMmary OF STATISTICAL TeSTS

\begin{tabular}{|c|c|c|c|}
\hline $\begin{array}{l}\text { Parameter } \\
\text { (1) }\end{array}$ & $\begin{array}{l}\text { Sample } \\
\text { (2) }\end{array}$ & $\begin{array}{c}\text { Mean } \\
(3)\end{array}$ & $\begin{array}{l}\text { Statistical Significance } \\
\text { (4) }\end{array}$ \\
\hline $\log \left(L_{\mathrm{CO}} / L_{B}\right) \ldots$ & $\begin{array}{l}\text { i) Normal galaxies } \\
\text { ii) All RSA Seyferts } \\
\text { iii) Type } 1 \text { RSA Seyferts } \\
\text { iv) Type } 2 \text { RSA Seyferts }\end{array}$ & $\begin{array}{l}-8.42 \\
-8.24 \\
-8.42 \\
-8.09\end{array}$ & $\begin{array}{c}\ldots 8 \%(\mathrm{i}) \\
58 \%(\mathrm{i}) \\
99.5 \%(\mathrm{i}) ; 90 \%(\mathrm{iii})\end{array}$ \\
\hline $\log \left(L_{\mathrm{CO}} / L_{\mathrm{FIR}}\right)$ & $\begin{array}{l}\text { i) Non-Seyferts } \\
\text { ii) Seyferts }\end{array}$ & $\begin{array}{l}-7.84 \\
-7.76\end{array}$ & $60 \%(\mathrm{i})$ \\
\hline $\log L_{\mathrm{FIR}} \ldots \ldots$ & $\begin{array}{l}\text { i) Control sample } \\
\text { ii) All RSA Seyferts } \\
\text { iii) Type } 1 \text { RSA Seyferts } \\
\text { iv) Type } 2 \text { RSA Seyferts }\end{array}$ & $\begin{array}{r}9.52 \\
9.92 \\
9.63 \\
10.13\end{array}$ & $\begin{array}{c}\ldots 9.7 \%(\mathrm{i}) \\
73 \%(\mathrm{i}) \\
>99.99 \%(\mathrm{i}) ; 99.3 \%(\mathrm{iii})\end{array}$ \\
\hline $\log \left(L_{\mathrm{CO}} / M_{\mathrm{HI}}\right) \ldots \ldots \ldots \ldots \ldots \ldots$ & $\begin{array}{l}\text { i) Normal Galaxies } \\
\text { ii) All Seyferts } \\
\text { iii) Type } 1 \text { Seyferts } \\
\text { iv) Type } 2 \text { Seyferts } \\
\text { v) All RSA Seyferts } \\
\text { vi) Type } 1 \text { RSA Seyferts } \\
\text { vii) Type } 2 \text { RSA Seyferts }\end{array}$ & $\begin{array}{r}-7.63 \\
\gtrsim-7.17 \\
\gtrsim-7.36 \\
\gtrsim-6.99 \\
\gtrsim-7.15 \\
-7.47 \\
\gtrsim-6.89\end{array}$ & $\begin{array}{c}\ldots \\
\gtrsim 98 \%(\mathrm{i}) \\
\gtrsim 65 \%(\mathrm{i}) \\
\gtrsim 99 \%(\mathrm{i}) \\
\gtrsim 97.5 \%(\mathrm{i}) \\
18 \%(\mathrm{i}) \\
\gtrsim 99.7 \%(\mathrm{i})\end{array}$ \\
\hline
\end{tabular}

Key to cols. (1) (4) follows: text.

Col. (1).- Parameter to be compared. Units are $L_{\mathrm{CO}}\left(\mathrm{K} \mathrm{km} \mathrm{s}^{-1} \mathrm{kpc}^{2}\right), L_{B}$ and $L_{\mathrm{FIR}}\left(L_{\odot}\right), M_{\mathrm{HI}}\left(M_{\odot}\right)$. See

Col. (2).-Samples to be compared (see text for details).

Col. (3).-Best estimate of the mean value of the parameter, computed using "survival analysis" techniques that explicitly utilize upper limits (see Feigelson and Nelson 1985$)$. For $\log \left(L_{\mathrm{CO}} / M_{\mathrm{HI}}\right)$ both lower and upper limits are present in all the Seyfert samples. The normal galaxy sample has only upper limits. Upper limits were treated as censored data in all cases, but, owing to the limitations of the statistical tests employed, the lower limits were treated as $\mathrm{H}$ i detections for the Seyfert samples. Thus, the quoted means and statistical significance levels for the Seyfert samples are only lower limits. These quoted values should be fairly accurate since only $\sim 10 \%$ of the Seyferts have lower limits on $\log \left(L_{\mathrm{CO}} / M_{\mathrm{H}}\right)$.

Col.(4).- Statistical significance of the difference between the corresponding mean listed in col. (3) and the mean for the comparison sample identified by the lower case Roman numeral in parentheses. These significance levels were computed using both generalized Wilcoxon and generalized Mantel-Cox tests. The mean of the two significance levels is tabulated.

radiation or dust reradiation of nonthermal light), we would expect that the Seyferts would systematically have an excess FIR luminosity at a given CO luminosity. Such is not the case. This strongly suggests that the FIR from most of these Seyferts has the same origin as in other galaxies-dust reradiation of starlight. Note that the conclusion probably does not apply to the near and mid-IR continuum $(\lambda \lesssim 20 \mu \mathrm{m})$ where the emission from Seyferts is likely to have a strong nuclear component (e.g., Miley, Neugebauer, and Soifer 1985). We will discuss this quantitatively and in detail in a future paper (Kirkpatrick et al. 1989).

We have seen above that the $\mathrm{CO}$ properties of Seyferts are strongly linked to their far-infrared properties, just as in the case of non-Seyferts. Thus, a comparison of the far-infrared properties of Seyferts and non-Seyferts is a valuable extension of the comparison of $\mathrm{CO}$ properties. The far-IR comparison has a decided advantage over its CO counterpart in that IRAS has provided us with a large and homogeneous data base concerning far-IR emission from galaxies. This allows us to conduct a more rigorous comparison of the Seyfert and normal galaxies, since we are better able to construct a comparison sample that accurately matches our Seyfert sample in all its essential properties.

Specifically, we have considered the 42 known Seyfert galaxies in the main catalog of the RSA and have compared them to a sample of 42 non-Seyfert galaxies chosen from the RSA to match the Seyfert sample in Hubble type (including the presence of bars), absolute blue magnitude, and distance. The rele- vant properties of the "RSA Seyfert" and "RSA control" samples are listed in Table 5.

The results of this comparison (summarized in Table 4 and illustrated in Fig. 7) strikingly confirm the results of the $\mathrm{CO}$ comparison. The RSA Seyferts as a whole are $\sim 2.5$ times more luminous in the FIR than are the RSA control galaxies (difference significant at the $99.7 \%$ confidence level).

However, it is entirely the type 2 RSA Seyferts that produce this effect. They are $\sim 4$ times more FIR-luminous than the RSA control galaxies and 3.2 times more FIR-luminous than the type 1 RSA Seyferts (difference significant at greater than $99.99 \%$ and $99.3 \%$ confidence levels, respectively). In contrast, the type 1 RSA Seyferts are not significantly more FIRluminous than the control galaxies. We emphasize that these differences are not due to any systematic differences in the distributions of absolute magnitude or Hubble type between the type 1 and type 2 RSA Seyferts. The differences in the infrared properties between the RSA Seyferts and normal galaxies will be discussed in greater detail in Kirkpatrick et al. (1989).

Edelson, Malkan, and Rieke (1987) have examined the infrared properties of the "CfA Seyfert sample" (Huchra and Berg 1988) which consists of 48 Seyfert galaxies with $m_{\mathrm{pg}}<14.5$ in a region of sky of $\Omega=2.66 \mathrm{sr}$ in extent. They reach several conclusions that can be compared to our results. First, they find that all of the type 2 Seyfert galaxies, but only half of the type 1 Seyferts exhibit clear evidence for thermal far-infrared emission whose origin they ascribe to dust heated by stars in the galaxy's 


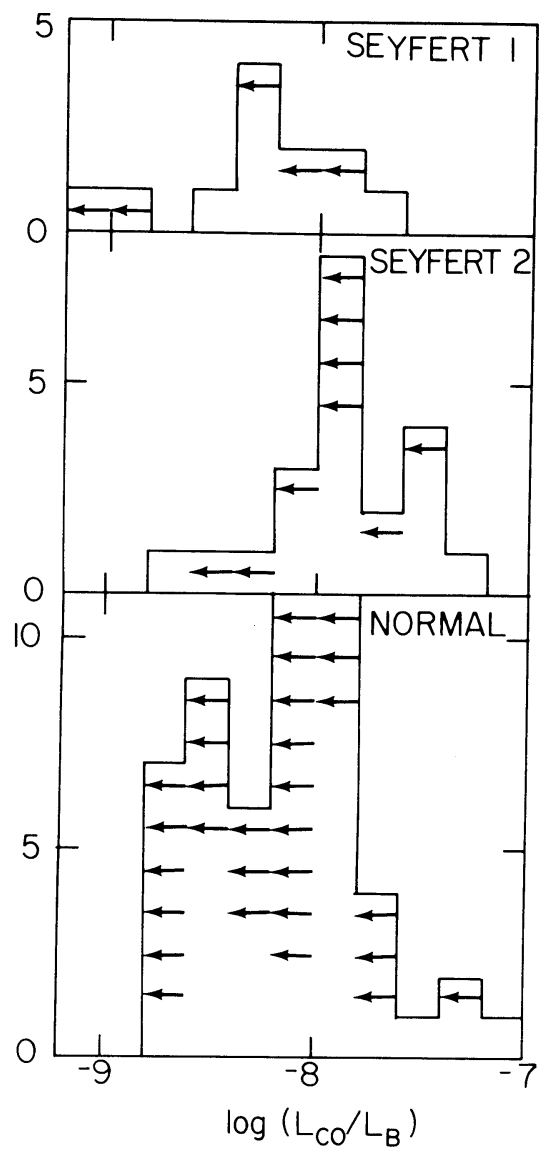

Fig. 4.- Histograms of the $\log$ of the ratio of CO-to-blue luminosity for type 1 Seyfert galaxies in the RSA (top), type 2 Seyfert galaxies in the RSA (middle), and normal galaxies (bottom). Upper limits (CO nondetections) are indicated by arrows. All galaxies plotted have Hubble types in the range SO/a to Sbc (see the text and Tables 2 and 3 for details). The type 2 RSA CO Seyferts have a significantly larger ( factor 2$)$ mean $\left(L_{\mathrm{Co}} / L_{B}\right)$ than the normal galaxies, but the type 1 RSA CO Seyferts do not (see text and Table 4).

disk. They also find that the far-infrared luminosity functions of the two Seyfert types are not significantly different from one another. Taken together, these two results then imply that the type 2 Seyferts are stronger sources of thermal ("disk") farinfrared emission than the type 1 Seyferts, while the latter have stronger nonthermal (nuclear) far-infrared emission.

These results are broadly consistent with our conclusions. While Edelson, Malkan, and Rieke do not report the factor of $\sim 3$ difference we found between the average total far-infrared luminosity of the RSA type 2 and type 1 Seyferts, we believe that this is largely a reflection of the greater nuclear (nonthermal) luminosity of the type 1 Seyferts in the CfA sample compared to those in our RSA sample. Many of the type 1 Seyferts in the CfA sample are dominated (in the optical) by the active nucleus: the ratios of the nucleus and galaxy optical luminosities are 5, 3.4, 1.4, and 2.3 for the type 1 CfA Seyferts Mrk 335, I Zw 1, Mrk 279, and Mrk 841, respectively (Malkan and Filippenko 1983; Malkan 1984; Yee 1983; Smith et al. 1986). In contrast, the type 1 RSA Seyferts have much weaker nonthermal optical nuclei: the ratios of nuclear-to- galaxy optical luminosities are only $0.03,0.02,0.09,0.15$, and 0.13 in NGC 3227, NGC 4151, NGC 5548, and NGC 7469, respectively (Malkan and Filippenko 1983).

Dahari and DeRobertis (1988) have also compared the farinfrared emission from type 1 and type 2 Seyferts in a large sample that (like the CfA sample) is populated with Seyferts having greater average nuclear luminosities than our RSA Seyferts. They find that while the average far-IR luminosities of the Seyfert 1 and 2 galaxies are similar in their sample, the Seyfert 2 galaxies have 2-3 times as much far-IR emission as type 1 Seyferts with the same optical nuclear luminosities (as measured by the [O III] $\lambda 5007$ luminosity of the narrow line region). This again supports a picture in which the extra-nuclear far-IR luminosity of the type 2 Seyferts is significantly greater than the type 1 Seyferts.

iii) Relationship to Star Formation

As reviewed recently by Wilson (1987) and Heckman (1988), a significant fraction of Seyfert galaxies exhibit evidence for star formation in regions exterior to the nucleus proper. We find that these Seyfert galaxies are precisely the Seyferts with the strongest $\mathrm{CO}$ emission. Below, we briefly summarize the evidence for star formation in Seyfert galaxies.

Narrow-band imaging and long-slit spectroscopy of the ionized gas in Seyfert galaxies demonstrate that there are two principal extranuclear components. The first has either a smooth morphology concentrated on the nucleus or a complex filamentary form. The emission-line ratios in this gas imply that it is probably photoionized by the diffuse high-energy continuum from the AGN, and the kinematics of the gas are sometimes complex with large noncircular motions (outflow?). This gas provides no direct evidence for star formation. The second component of ionized gas is knotty or clumpy in morphology and is associated with spiral arms or circumnuclear rings. The emission-line ratios are consonant with photoionization by normal OB stars and the kinematics of the gas imply that it is part of the rotating disk of the galaxy. This component appears to be a population of giant $\mathrm{H}$ II regions and as such is a good tracer of star formation.

The nonthermal radio sources in Seyferts can likewise be divided into two morphological classes. The first are the "linear" sources (jetlike, double, or colinear triple), and these are almost certainly powered by the AGN. The second and less common class are the "diffuse" sources. These are associated with the regions of star formation delineated in the optical (see above) and may be powered by the young stellar population.

Seyfert galaxies also exhibit considerable diversity in their $\mathrm{mid} /$ far-infrared (M/FIR) spectral energy distributions (e.g., Miley, Neugebauer, and Soifer 1985; Edelson, Malkan, and Rieke 1987). As discussed in Kirkpatrick, Heckman, and Wilson (1989), many Seyfert galaxies (particularly those of type 2) have M/FIR colors that are indistinguishable from those of the Markarian starburst galaxies. Other Seyferts have M/FIR colors like those of quasars. Wilson (1987), Edelson, Malkan, and Rieke (1987), and Edelson and Malkan (1986) have shown that the optical and radio evidence for star formation discussed above correlates well with the M/FIR colors (i.e., those Seyferts with starburst-like colors have diffuse radio sources and extranuclear giant $\mathrm{H}$ II regions).

In a number of Seyferts there is clear evidence that the midinfrared-emitting region $(\lambda \sim 10 \mu \mathrm{m})$ is spatially extended on scales $\gtrsim 1 \mathrm{kpc}$, representing strong evidence for a spatially distributed heat source, almost certainly young stars (see 

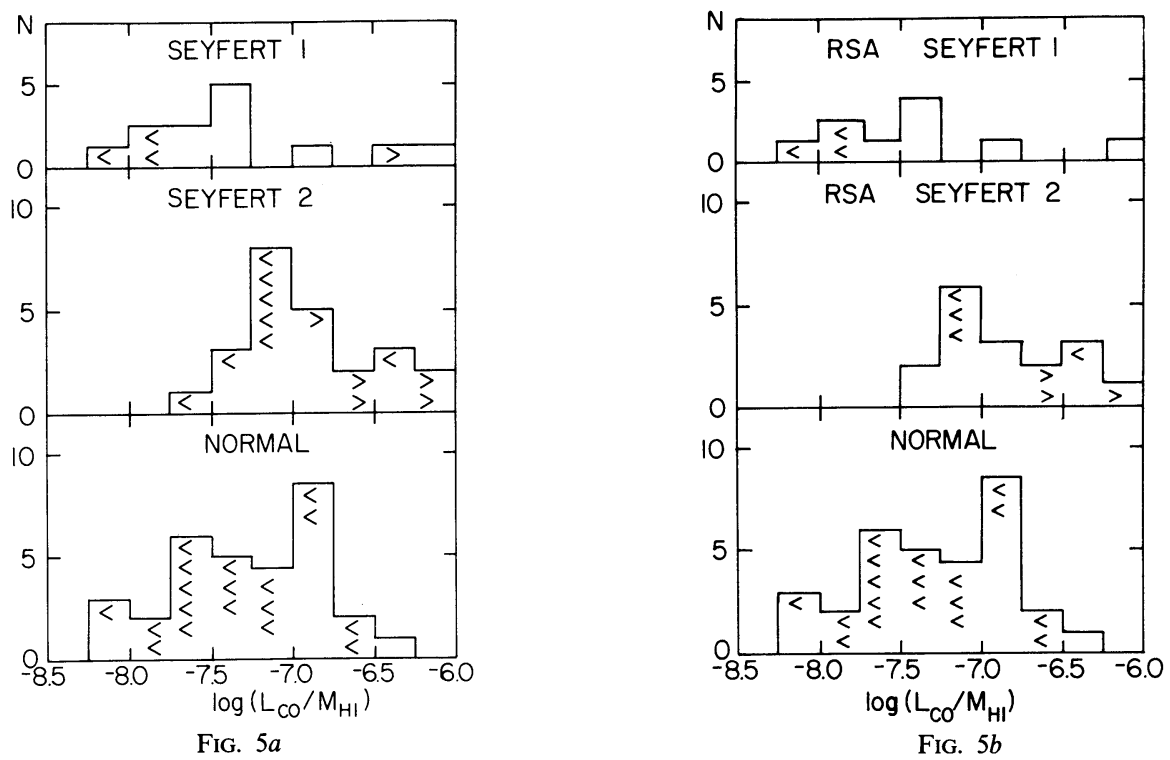

FIG. 5. $-(a)$ Histograms of the $\log$ of the ratio of $\mathrm{CO}$ luminosity $\left(\mathrm{K} \mathrm{km} \mathrm{s}^{-1} \mathrm{kpc}^{2}\right)$ to $\mathrm{H}$ I mass $\left(M_{\odot}\right)$ for all type 1 Seyfert galaxies (top), all type 2 Seyfert galaxies (middle), and normal disk galaxies (bottom). Both upper and lower limits (indicated by left-facing and right-facing arrows) exist in this ratio. (b) As in (a) except that only Seyfert galaxies in the Revised Shapley-Ames Catalog (RSA) are plotted.

Edelson, Malkan, and Rieke 1987; Wynn-Williams, Becklin, and Scoville 1985). In Table 6 and its associated notes we summarize the optical, radio, and infrared evidence for star formation for the 13 Seyferts with the largest CO luminosities $\left[L(C O)>1000 \mathrm{~K} \mathrm{~km} \mathrm{~s}^{-1} \mathrm{kpc}^{2}\right]$. All the CO-bright Seyferts for which the relevant data exist appear to be undergoing extensive star formation.

\section{iv) Relation to the Nonthermal Radio Power}

A correlation between the $\mathrm{CO}$ luminosity and nonthermal radio continuum power for galaxies has been found by Rickard, Turner, and Palmer (1985) and Stark et al. (1986). It is interpreted as evidence that both parameters are governed by the extreme Population I component of a galaxy. The origin of radio continuum emission in Seyfert galaxies is variously ascribed to processes fueled by the compact active nucleus (e.g., Wilson and Ulvestad 1982) or to processes fueled by the evolution of young, massive stars (e.g., Condon et al. 1982). It is therefore quite instructive to consider the relation between the $\mathrm{CO}$ and nonthermal radio continuum luminosities for a large sample of Seyfert galaxies.

Figure $8 a$ is a plot of the CO luminosity against the monochromatic nonthermal radio power at $1.4 \mathrm{GHz}$ for the Seyfert galaxies in Table 2. The diagram suggests that the two variables are related, and a generalized Kendall's $\tau$-test (which utilizes both the detections and upper limits in either variable) indicates that the correlation is significant at the $99.9 \%$ confidence level (see Isobe, Feigelson, and Nelson 1986, and references therein). A standard nonparametric K-S test using only the galaxies with detected $\mathrm{CO}$ and $1.4 \mathrm{GHz}$ emission confirms the strongly significant correlations (greater than $99.9 \%$ confidence level).

In order to interpret this correlation, we have constructed a similar plot in Figure $8 b$ for a large sample of non-Seyfert galaxies. This sample comprises the $\mathrm{CO}$ surveys of normal galaxies conducted by Stark et al. (1986) and Stark, Elmegreen, and Chance (1987) and of infrared-bright galaxies by Sanders and Mirabel (1985), Sanders et al. (1986), and Young et al. $(1984,1986)$. We have excluded any known Seyfert galaxies from these samples. The non-Seyferts show a very strong correlation between $L_{\mathrm{CO}}$ and $P_{1.4}$ (greater than $99.99 \%$ confidence). Using Schmitt's (1985) two-dimensional KaplanMeier estimator, we find that the maximum-likelihood linear regression between the two variables is $\log L_{\mathrm{CO}}=0.74 \log P_{1.4}$ - 13.85. A nonparametric K-S test using only the $\mathrm{CO}$ and 1.4 $\mathrm{GHz}$ detections also yields a significance level greater than $99.9 \%$ for the correlation.

Relative to this regression line (see Fig. $8 b$ ), we find that 24 of the non-Seyferts lie to the lower right, 38 lie to the upper left, and 30 nondetections have an uncertain location relative to the line. For the Seyfert sample in Figure $8 a, 27$ lie to the lower right of the same line, only 11 to the upper left, and 13 nondetections have an uncertain location relative to the line. The differences in these two distributions (Seyfert vs. non-Seyfert) is significant at better than the $99.7 \%$ confidence level (using a $\chi^{2}$ contingency table analysis). This result demonstrates that for $a$ given CO luminosity, Seyfert galaxies have a higher average nonthermal radio power.

The Seyfert galaxies having the largest excess radio power for their CO luminosity are NGC 1275, NGC 2110, NGC 2992, NGC 4151, NGC 4388, NGC 5506, NGC 7682, Mrk 3, Mrk 463, and MCG 8-11-11. Except for NGC 7682 (for which no high-resolution radio map exists), all these galaxies show clear morphological evidence that a compact active nucleus plays a dominant role in fueling the radio emission (i.e., they all exhibit jets and/or double or colinear triple radio sources). On the other hand, many Seyfert galaxies have radio powers commensurate with those of normal galaxies of similar CO luminosities. We will discuss our interpretation of the above results in $\S \mathrm{IV} b$ below.

\section{b) The CO Kinematics}

Given the nature of the existing data base, we have taken a simple statistical approach to our investigation of the CO kinematics. For all the Seyferts with detected $\mathrm{CO}$ emission, we have 
TABLE 5

ReVISED ShaPLeY-AMES SAMPLES

\begin{tabular}{|c|c|c|c|c|c|c|c|}
\hline $\begin{array}{r}\text { Gal } \\
(1\end{array}$ & $\begin{array}{l}\operatorname{laxy} \\
1)\end{array}$ & $\begin{array}{c}\text { Type } \\
(2)\end{array}$ & $\begin{array}{c}B_{T}^{0, i} \\
(3)\end{array}$ & $\begin{array}{c}M_{B_{T}}^{0, i} \\
(4)\end{array}$ & $\begin{array}{c}v_{0} \\
(5)\end{array}$ & $\begin{array}{c}L_{\mathrm{FIR}} \\
(6)\end{array}$ & $\begin{array}{c}\text { Subsample } \\
(7)\end{array}$ \\
\hline NGC 175 & & SBab & 12.32 & -21.29 & 3962 & 0.48 & $\mathrm{C}$ \\
\hline NGC 278 & $\ldots \ldots \ldots \ldots \ldots$ & Sbc & 10.85 & -19.62 & 932 & 0.63 & $\mathrm{C}$ \\
\hline NGC $782 \ldots$ & $\ldots \ldots \ldots \ldots \ldots$ & $\mathrm{SBb}$ & 12.36 & -22.07 & 5584 & 3.0 & $\mathrm{C}$ \\
\hline NGC 788 . & $\ldots \ldots \ldots \ldots \ldots$ & $\mathrm{Sa}$ & 13.00 & -20.76 & 4238 & 0.22 & $\mathrm{~S} 2$ \\
\hline NGC 908 & $\ldots \ldots \ldots$ & Sc & 10.33 & -21.27 & 1563 & 1.7 & $\mathrm{C}$ \\
\hline NGC 1068 & $\ldots \ldots \ldots \ldots$ & $\mathrm{Sb}$ & 9.03 & -22.05 & 1234 & 7.4 & $\mathrm{~S} 2$ \\
\hline NGC 1232 & n......... & $\mathrm{Sc}$ & 10.18 & -21.69 & 1775 & 1.2 & $\mathrm{C}$ \\
\hline NGC 1275 & ........... & $\mathrm{E}$ & 11.91 & -22.39 & 5433 & 4.8 & S2 \\
\hline NGC 1291 & ........... & $\mathrm{SBa}$ & 9.17 & -20.80 & 738 & 0.05 & $\mathrm{C}$ \\
\hline NGC 1350 & n......... & $\mathrm{Sa}$ & 11.02 & -20.50 & 1862 & 0.18 & $\mathrm{C}$ \\
\hline NGC 1358 & $\ldots \ldots \ldots \ldots$ & $\mathrm{Sa}$ & 12.95 & -20.75 & 4114 & 0.28 & S2 \\
\hline NGC 1365 & ........... & $\mathrm{Sb}$ & 9.45 & -22.07 & 1562 & 6.8 & $\mathrm{~S} 2$ \\
\hline NGC 1386 & ........... & $\mathrm{Sa}$ & 11.08 & -20.48 & 826 & 0.41 & $\mathrm{~S} 2$ \\
\hline NGC 1415 & $\ldots \ldots \ldots \ldots$ & $\mathrm{SBa}$ & 11.02 & -19.80 & 1526 & 0.46 & $\mathrm{C}$ \\
\hline NGC 1512 & n......... & $\mathrm{SBb}$ & 10.77 & -19.26 & 760 & 0.07 & $\mathrm{C}$ \\
\hline NGC 1566 & ........... & Sc & 9.79 & -21.41 & 1305 & 1.5 & S1 \\
\hline NGC 1638 & .......... & $\mathrm{Sa}$ & 12.64 & -20.58 & 3308 & $\lesssim 0.2$ & $\mathrm{C}$ \\
\hline NGC 1667 & n......... & Sc & 12.26 & -21.66 & 4562 & 4.9 & $\mathrm{~S} 2$ \\
\hline NGC 2268 & ........... & Sbc & 11.63 & -20.95 & 2458 & 1.1 & $\mathrm{C}$ \\
\hline NGC 2523 & $\ldots \ldots \ldots \ldots$ & $\mathrm{SBb}$ & 11.86 & -21.57 & 3638 & 0.57 & $\mathrm{C}$ \\
\hline NGC 2551 & $\ldots \ldots$ & $\mathrm{Sab}$ & 12.31 & -20.29 & 2484 & 0.09 & $\mathrm{C}$ \\
\hline NGC 2639 & n......... & $\mathrm{Sa}$ & 11.90 & -21.30 & 3270 & 0.94 & $\mathrm{~S} 1$ \\
\hline NGC 2782 & ............ & $\mathrm{Sa}$ & 11.50 & -21.18 & 2574 & 1.9 & $\mathrm{C}$ \\
\hline NGC 2787 & ........... & $\mathrm{SBO} / \mathrm{a}$ & 11.66 & -18.56 & 828 & $\sim 0.01$ & $\mathrm{C}$ \\
\hline NGC 2811 & $\ldots \ldots \ldots \ldots$ & $\mathrm{Sa}$ & 11.54 & -20.87 & 2279 & 0.11 & $\mathrm{C}$ \\
\hline NGC 2832 & $\ldots \ldots \ldots \ldots$ & $\mathrm{E}$ & 12.39 & -22.44 & 6935 & 0.82 & $\mathrm{C}$ \\
\hline NGC 2992 & ........... & $\mathrm{Sa}$ & 11.61 & -20.48 & 1963 & 2.1 & $\mathrm{~S} 2$ \\
\hline NGC 3081 & $\ldots \ldots \ldots \ldots$ & $\mathrm{Sa}$ & 12.50 & -19.79 & 2148 & $?$ & $\mathrm{~S} 2$ \\
\hline NGC 3227 & n......... & $\mathrm{Sb}$ & 10.92 & -19.92 & 1102 & 0.41 & S1 \\
\hline NGC 3241 & ........... & $\mathrm{Sb}$ & 12.77 & -19.92 & 2584 & 0.37 & $\mathrm{C}$ \\
\hline NGC 3275 & ........... & SBab & 11.57 & -21.40 & 2944 & 0.47 & $\mathrm{C}$ \\
\hline NGC 3281 & $\ldots \ldots \ldots \ldots$ & $\mathrm{Sa}$ & 11.96 & -21.23 & 3255 & 2.0 & $\mathrm{~S} 2$ \\
\hline NGC 3329 & $\ldots \ldots \ldots \ldots$ & $\mathrm{Sab}$ & 12.20 & -20.04 & 2102 & 0.13 & $\mathrm{C}$ \\
\hline NGC 3516 & $\ldots \ldots \ldots \ldots$ & SO & 12.34 & -20.55 & 2838 & 0.38 & $\mathrm{~S} 1$ \\
\hline NGC 3571 & ........... & $\mathrm{Sa}$ & 11.78 & -21.58 & 3520 & 0.09 & $\mathrm{C}$ \\
\hline NGC 3628 & $\ldots \ldots \ldots \ldots$ & Sbc & 9.49 & -20.42 & 719 & 0.96 & $\mathrm{C}$ \\
\hline NGC 3783 & $\ldots \ldots \ldots \ldots$ & $\mathrm{Sa}$ & 12.68 & -19.93 & 2494 & 0.61 & S1 \\
\hline NGC 3865 & ............ & $\mathrm{Sab}$ & 12.26 & -22.06 & 5491 & 0.40 & $\mathrm{C}$ \\
\hline NGC 3982 & ........... & Sbc & 11.59 & -19.42 & 1195 & 0.35 & $\mathrm{~S} 2$ \\
\hline NGC 4051 & $\ldots \ldots \ldots \ldots$ & Sbc & 10.56 & -19.43 & 746 & 0.20 & S1 \\
\hline NGC 4151 & ............ & $\mathrm{Sab}$ & 10.53 & -20.05 & 978 & 0.16 & $\mathrm{~S} 1$ \\
\hline NGC 4157 & ........... & Sbc & 10.73 & -19.55 & 852 & 0.51 & $\mathrm{C}$ \\
\hline NGC 4224 & n......... & $\mathrm{Sa}$ & 12.04 & -21.40 & 2442 & 0.06 & $\mathrm{C}$ \\
\hline NGC 4235 & $\ldots \ldots \ldots \ldots$ & $\mathrm{Sa}$ & 12.00 & -20.51 & 2380 & 0.06 & S1 \\
\hline NGC 4260 & n......... & $\mathrm{SBa}$ & 12.30 & -19.61 & 1806 & 0.05 & $\mathrm{C}$ \\
\hline NGC 4388 & n.......... & $\mathrm{Sab}$ & 10.65 & -20.17 & 2369 & 0.32 & $\mathrm{~S} 2$ \\
\hline NGC 4394 & ............ & $\mathrm{SBb}$ & 11.28 & -19.54 & 853 & 0.03 & $\mathrm{C}$ \\
\hline NGC 4507 & $\ldots \ldots \ldots \ldots$ & $\mathrm{Sab}$ & 12.09 & -21.08 & 3226 & 1.4 & $\mathrm{~S} 2$ \\
\hline NGC 4593 & $\ldots \ldots \ldots \ldots$ & $\mathrm{Sb}$ & 11.15 & -21.47 & 2505 & 0.79 & S1 \\
\hline NGC 4639 & $\ldots \ldots \ldots \ldots$ & $\mathrm{Sb}$ & 11.57 & -19.25 & 860 & 0.06 & $\mathrm{~S} 1$ \\
\hline NGC 4654 & $\ldots \ldots \ldots \ldots$ & $\mathrm{SBc}$ & 10.68 & -20.14 & 926 & 0.42 & $\mathrm{C}$ \\
\hline NGC 4941 & $\ldots \ldots \ldots \ldots$ & $\mathrm{Sab}$ & 11.15 & -19.19 & 878 & 0.36 & $\mathrm{~S} 2$ \\
\hline NGC 5033 & n.......... & Sbc & 10.11 & -20.28 & 897 & 0.53 & $\mathrm{~S} 1$ \\
\hline NGC 5064 & $\ldots \ldots \ldots \ldots$ & $\mathrm{Sa}$ & 11.39 & -21.39 & 2700 & 1.0 & $\mathrm{C}$ \\
\hline NGC 5135 & .......... & $\mathrm{Sb}$ & 11.91 & -21.67 & 3906 & 9.0 & $\mathrm{~S} 2$ \\
\hline NGC 5194 & $\ldots \ldots \ldots \ldots$ & $\mathrm{Sbc}$ & 8.57 & -20.72 & 541 & 0.97 & $\mathrm{~S} 2$ \\
\hline NGC 5248 & $\ldots \ldots \ldots \ldots$ & Sbc & 10.42 & -20.31 & 1049 & 0.84 & $\mathrm{C}$ \\
\hline NGC 5273 & $\ldots \ldots \ldots \ldots$ & $\mathrm{SO} / \mathrm{a}$ & 12.42 & -18.35 & 1070 & 0.04 & $\mathrm{~S} 1$ \\
\hline NGC 5347 & $\ldots \ldots \ldots \ldots$ & $\mathrm{Sb}$ & 12.86 & -19.66 & 2394 & 0.28 & $\mathrm{~S} 2$ \\
\hline NGC 5351 & $\ldots \ldots \ldots \ldots$ & $\mathrm{SBb}$ & 12.25 & -21.19 & 3663 & 1.1 & $\mathrm{C}$ \\
\hline NGC 5364 & $\ldots \ldots \ldots \ldots$ & $\mathrm{Sc}$ & 10.64 & -20.27 & 1140 & 0.24 & $\mathrm{C}$ \\
\hline NGC 5365 & $\ldots \ldots \ldots \ldots$ & SBO & 11.90 & -20.45 & 2209 & 0.04 & $\mathrm{C}$ \\
\hline NGC 5427 & $\ldots \ldots \ldots \ldots$ & Sbc & 11.76 & -20.91 & 2565 & 2.3 & $\mathbf{S} 2$ \\
\hline NGC 5448 & ........... & $\mathrm{Sa}$ & 11.35 & -20.91 & 2121 & 0.33 & $\mathrm{C}$ \\
\hline NGC 5548 & $\ldots \ldots \ldots \ldots$ & $\mathrm{Sa}$ & 12.90 & -21.20 & 4964 & 0.86 & S1 \\
\hline NGC 5614 & n.......... & $\mathrm{Sa}$ & 12.02 & -21.57 & 3915 & 1.1 & $\mathrm{C}$ \\
\hline NGC 5643 & $\ldots \ldots \ldots \ldots$ & Sc & 10.19 & -20.32 & 947 & 0.67 & $\mathbf{S} 2$ \\
\hline NGC 5728 & $\ldots \ldots \ldots \ldots$ & $\mathrm{Sb}$ & 11.27 & -21.59 & 2800 & 2.2 & $\mathrm{~S} 2$ \\
\hline NGC 5905 & $\ldots \ldots \ldots \ldots$ & $\mathrm{SBb}$ & 11.77 & -21.60 & 3544 & 1.4 & $\mathrm{C}$ \\
\hline NGC 6221 & $\ldots \ldots \ldots \ldots$ & Sbc & 10.47 & -20.67 & 1270 & 2.5 & $\mathrm{C}$ \\
\hline
\end{tabular}


TABLE 5-Continued

ReVised ShaPley-Ames SAMPLES

\begin{tabular}{|c|c|c|c|c|c|c|}
\hline $\begin{array}{c}\text { Galaxy } \\
\text { (1) }\end{array}$ & $\begin{array}{c}\text { Type } \\
(2)\end{array}$ & $\begin{array}{c}B_{T}^{0, i} \\
(3)\end{array}$ & $\begin{array}{c}M_{B_{T}}^{0, i} \\
(4)\end{array}$ & $\begin{array}{l}v_{0} \\
(5)\end{array}$ & $\begin{array}{c}L_{\text {FIR }} \\
(6)\end{array}$ & $\begin{array}{c}\text { Subsample } \\
\text { (7) }\end{array}$ \\
\hline NGC 6300 & $\mathrm{Sb}$ & 9.96 & -20.44 & 901 & 0.52 & S2 \\
\hline NGC 6814 & Sbc & 11.37 & -20.33 & 1647 & 0.66 & $\mathrm{~S} 1$ \\
\hline NGC $6890 \quad \ldots \ldots \ldots \ldots$ & $\mathrm{Sab}$ & 12.35 & -20.18 & 2401 & 0.80 & $\mathrm{~S} 2$ \\
\hline NGC $6951 \quad \ldots \ldots \ldots \ldots$ & $\mathrm{SBb}$ & 11.31 & -20.48 & 1710 & 1.8 & $\mathrm{C}$ \\
\hline IC $5063 \ldots \ldots \ldots \ldots$ & $\mathrm{SO} / \mathrm{a}$ & 13.14 & -20.07 & 3289 & 1.5 & $\mathbf{S} 2$ \\
\hline NGC $7007 \ldots$ & $\mathrm{SO} / \mathrm{a}$ & 12.92 & -19.99 & 2866 & 0.08 & $\mathrm{C}$ \\
\hline IC $5135 \ldots \ldots$. & $\mathrm{Sa}$ & 12.64 & -21.39 & 4808 & 12 & S2 \\
\hline NGC $7213 \ldots$ & $\mathrm{Sa}$ & 10.72 & -21.08 & 1720 & 0.36 & $\mathrm{~S} 1$ \\
\hline NGC $7314 \ldots \ldots \ldots$ & Sc & 11.11 & -20.38 & 1491 & 0.58 & $\mathrm{~S} 1$ \\
\hline NGC $7469 \quad \ldots \ldots \ldots \ldots$ & $\mathrm{Sab}$ & 11.95 & -22.22 & 5115 & 18 & $\mathrm{~S} 1$ \\
\hline NGC $7582 \ldots \ldots \ldots$ & $\mathrm{Sab}$ & 10.60 & -20.87 & 1475 & 3.4 & $\mathrm{~S} 2$ \\
\hline NGC $7690 \quad \ldots \ldots \ldots \ldots$ & $\mathrm{Sab}$ & 11.82 & -19.36 & 1291 & 0.12 & C \\
\hline NGC $7716 \ldots \ldots \ldots$ & $\mathrm{Sab}$ & 12.43 & -20.38 & 2735 & 0.21 & $\mathrm{C}$ \\
\hline NGC $7782 \ldots \ldots \ldots \ldots$ & $\mathrm{Sb}$ & 12.36 & -22.00 & 5584 & 1.4 & $\mathrm{C}$ \\
\hline
\end{tabular}

Key to cols. (2)-(7) follows:

Col. (2).-Morphological type from the RSA.

Col. (3).-Apparent blue magnitude in the $B_{T}$ system corrected for Galactic and internal absorption (from the RSA).

Col. (4).-Absolute blue magnitude in the $B_{T}$ system corrected for galactic and internal absorption, assuming that $H_{0}=75 \mathrm{~km} \mathrm{~s}^{-1} \mathrm{Mpc}^{-1}$, and using the velocity in col. (5). The exceptions are NGC 1350, NGC 1365, NGC 1386 (assumed to be in the Fornax cluster at a distance of $20 \mathrm{Mpc}$ ), and NGC 4388, NGC 4394, and NGC 4639 (assumed to be in the Virgo cluster at a distance of $14 \mathrm{Mpc}$ ).

Col. (5).-Corrected recession velocity $\left(\mathrm{km} \mathrm{s}^{-1}\right)$ relative to the centoid of the Local Group (from the RSA).

Col. (6).-Far-infrared luminosity in units of $10^{10} L_{\odot}$, calculated using the far-infrared flux (as defined by Lonsdale et al. 1985) and assuming $H_{0}=75 \mathrm{~km} \mathrm{~s}^{-1} \mathrm{Mpc}^{-1}$.

Col. (7).-Subsamples are "C" (control sample), "S1" (type 1 Seyfert sample), and "S2" (type 2 Seyfert sample).

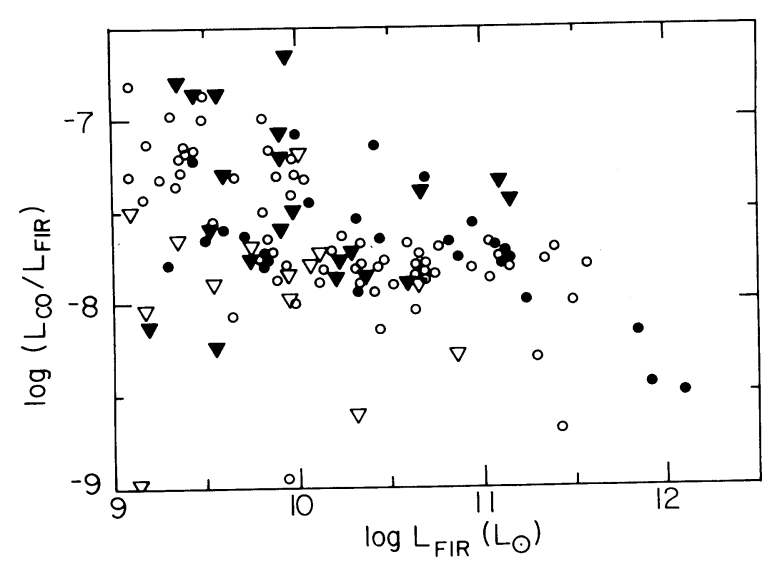

Fig. 6.-The log of the far-infrared luminosity (in $L_{\odot}$ ) is plotted against the $\log$ of the ratio of the $\mathrm{CO}$ luminosity (in $\mathrm{K} \mathrm{km} \mathrm{s} \mathrm{km}^{-1} \mathrm{kpc}^{2}$ ) and the far-infrared luminosity for Seyfert galaxies (solid points) and non-Seyfert galaxies (open points) $\mathrm{CO}$ detections are indicated by circles and nondetections (upper limits) by triangles. The Seyfert $\mathrm{CO}$ and IR data are taken from Tables 2 and 5. For the non-Seyferts, the CO data are from Stark et al. (1986), Sanders and Mirabel (1985), Sanders et al. (1986), and Young et al. $(1984,1986)$, and the IR data are from the IRAS Point Source Catalog. The Seyferts and non-Seyferts follow the same relationship between $L_{\mathrm{CO}}$ and $L_{\mathrm{FIR}}$.

assembled the existing data regarding the global kinematics of other major constituents of Seyfert galaxies: the H I (probed with the $\lambda-21 \mathrm{~cm}$ line), the ionized gas in the narrow line region (the NLR, as probed with the [O III] $\lambda 5007$ line), and the near-nuclear stellar population (as probed with the central stellar velocity dispersion). These data are summarized in Table 7, and are further described in the associated notes.

The first conclusion drawn from these data is that much of the $\mathrm{CO}$ is likely to be arrayed in a rotating disk that is coplanar with the large-scale galaxy/H I disk. The evidence is illustrated in Figures 9 and 10 and is quantified in Table 8. First, the observed CO line widths clearly correlate with the galaxy inclination. This correlation is surprisingly good in view of the likely range in the intrinsic line widths and the uncertainties in the inferred inclinations (see notes to Table 7). Second, the CO line widths correlate with the $\mathrm{H}$ I line widths. This correlation is equally good between the measured $\mathrm{CO}$ and $\mathrm{H}$ I line widths and the $\mathrm{CO}$ and $\mathrm{H} \mathrm{I}$ line widths corrected for inclination (Figs. $10 a, 10 b$ and Table 8).

While the above might suggest that the $\mathrm{CO}$ resides primarily in the large-scale galaxy disk along with $\mathrm{H} \mathrm{I}$, Figure $11 a$ demonstrates (as was first noticed by Blitz, Mathieu, and Bally 1986 for a much smaller sample of Seyfert galaxies) that the CO line widths correlate weakly with the nuclear $[\mathrm{O}$ III $] \lambda 5007$ line widths. The correlation improves dramatically when the $\mathrm{CO}$ (but not the [O III]) line widths are corrected for inclination (Fig. 11b). Indeed, the ratio of the CO-to-[O III] line widths clearly correlates with the sine of the estimated galaxy inclination (Fig. 12 and Table 8). Moreover, the correlations between $W_{\mathrm{CO}}$ and $W_{\mathrm{O} \text { III }}$ and between $W_{\mathrm{CO}} / \sin i$ and $W_{\mathrm{O} \text { III }}$ are both stronger than the corresponding correlations between $W_{\mathrm{HI}}$ and $W_{\mathrm{O} \text { III }}$ and between $W_{\mathrm{H}} / \sin i$ and $W_{\mathrm{O} \text { III }}$ (Table 8). This suggests that the NLR kinematics are more closely related to the molecular gas than to the atomic gas. Since the [O III] lines arise in the NLR in the central few hundred parsecs of the galaxy, the $\mathrm{CO}$ versus [O $\mathrm{III}]$ line width correlation suggests that a significant fraction of the CO may also be circumnuclear in scale. High angular resolution observations of the $\mathrm{CO}$ in a moderately large sample of Seyferts are essential to test this hypothesis (see Heckman 1989 for a progress report).

Finally, Figures $13 a$ and $13 b$ illustrate the relationship between the CO line widths and stellar velocity dispersions. 
TABLE 6

EVIDENCE FOR STAR FORMATION IN CO-BRIGHT SEYFERTS

\begin{tabular}{|c|c|c|c|c|c|}
\hline $\begin{array}{l}\text { Galaxy } \\
\text { (1) }\end{array}$ & $\begin{array}{l}L_{\mathrm{CO}} \\
(2)\end{array}$ & $\begin{array}{l}\text { IR Colors } \\
\text { (3) }\end{array}$ & $\begin{array}{l}\text { Radio Morphology } \\
\text { (4) }\end{array}$ & $\begin{array}{l}\text { Extended IR } \\
\text { (5) }\end{array}$ & $\begin{array}{l}\text { H II Regions } \\
\text { (6) }\end{array}$ \\
\hline Mrk $938 \ldots$ & 2300 & $S$ & & $?$ & $?$ \\
\hline NGC $1068 \ldots \ldots \ldots \ldots$ & 1300 & Q & $\mathrm{L}+\mathrm{D}$ & Y & $\mathrm{Y}$ \\
\hline NGC $1365 \quad \ldots \ldots \ldots \ldots$ & 1400 & $\mathrm{~s}$ & D & $\mathbf{Y}$ & $\mathbf{Y}$ \\
\hline NGC $1667 \quad \ldots \ldots \ldots \ldots$ & 2300 & $S$ & & $?$ & $\mathrm{Y}$ \\
\hline Mrk $10 \ldots \ldots \ldots \ldots \ldots$ & 1900 & $\mathrm{Q} / \mathrm{S}$ & & $?$ & $\mathrm{Y}$ \\
\hline Mrk $231 \ldots \ldots \ldots \ldots \ldots$ & 4100 & s & $\mathrm{D}$ & $\mathbf{N}$ & $?$ \\
\hline NGC $5135 \ldots \ldots$ & 2400 & $\mathrm{~S}$ & $?$ & $?$ & \\
\hline Mrk $266 \ldots \ldots \ldots \ldots \ldots$ & 2700 & $\mathrm{~S}$ & & $?$ & ? \\
\hline Mrk $273 \ldots \ldots \ldots \ldots \ldots$ & 4000 & $S$ & $?$ & $\dot{\mathrm{Y}}$ & $?$ \\
\hline Arp $220 \ldots \ldots \ldots \ldots$ & 2900 & $\mathrm{~S}$ & $\mathbf{L}$ & $\mathbf{N}$ & ? \\
\hline IC $5135 \ldots \ldots \ldots \ldots \ldots$ & 2400 & S & & $?$ & Y \\
\hline NGC $7469 \quad \ldots \ldots \ldots \ldots$ & 1800 & $\mathrm{~S}$ & $\mathrm{D}$ & $\dot{\mathrm{Y}}$ & $\mathrm{Y}$ \\
\hline NGC $7674 \ldots \ldots \ldots \ldots$ & 2300 & Q & & $?$ & \\
\hline
\end{tabular}

Key to cols. (2)-(6) follows:

Col. (2).-CO luminosity from Table $2\left(\mathrm{~K} \mathrm{~km} \mathrm{~s}^{-1} \mathrm{kpc}^{2}\right)$. When more than one $L_{\mathrm{CO}}$ is listed in Table 2, the quoted value is a log-average.

Col. (3). Classification of the mid/far-IR colors from the 25,60 , and $100 \mu \mathrm{m} I R A S$ data. " $\mathrm{S}$ " means the IR colors are similar to those of starburst galaxies from Balzano's 1983 sample, while "Q" means the IR colors are similar to those of quasars (see Neugebauer et al. 1986 and Miley, Neugebauer, and Soifer 1985).

Col. (4).- Radio morphological type, where " $D$ " denotes diffuse radio sources and " $L$ " denotes linear radio sources. An open space indicates that the radio source has never been mapped (never observed or never resolved spatially), a "?" indicates that the radio morphology is ambiguous. See Wilson and Heckman 1985 or Wilson (1987) for further discussion.

Col. (5).- " Y" means that the mid-infrared emission (10-12 $\mu \mathrm{m})$ is spatially resolved, favoring a distributed dust-heating source (i.e., young stars). See Edelson, Malkan, and Rieke 1987 and Rodriguez-Espinosa, Rudy, and Jones 1986

Col. (6).- " $Y$ " indicates that regions whose morphology and/or emission-line ratios are consistent with giant $\mathrm{H}$ II regions have been detected in the extranuclear region of the Seyfert galaxy. An open space indicates that no adequate data exist for assessing whether giant $\mathrm{H}$ II regions exist; " ?" indicates that the existing data are ambiguous. Data used are from Wilson et al. 1986, Baldwin, Wilson, and Whittle 1987, Phillips et al. 1983, Balick and Heckman 1985, or are our unpublished images and spectra.

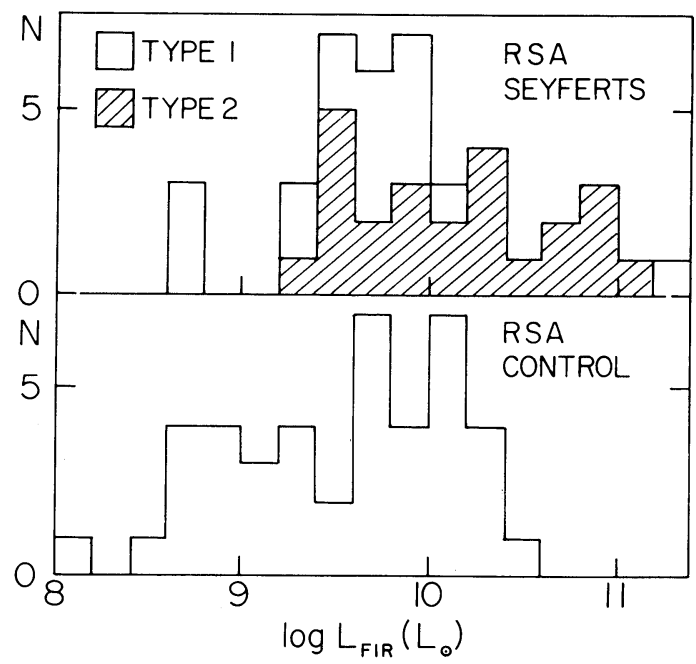

FIG. 7.-Histograms of the log of the far-infrared luminosities of the Seyfert galaxies in the Revised Shapley-Ames Catalog (top) and a control sample of non-Seyfert galaxies having similar Hubble types, distances, and absolute magnitudes (bottom). The samples and relevant data are listed and discussed in Table 5. The Seyfert sample as a whole is 2.5 times more luminous in the mean (see Table 4$)$. This is due almost entirely to the type 2 Seyferts ( $\sim 4$ times more luminous than the control sample).
The sample is so small (seven members) that only tentative conclusions can be drawn. It does appear that the line widths are correlated and that the correlation improves when the $\mathrm{CO}$ line widths are corrected for inclination. This is just as expected, since the stellar velocity dispersions represent the rms line-of-sight velocities in the inner bulge and should not be strongly aspect-angle-dependent (provided rotation is not dominant).

\section{c) Very High Velocity $\mathrm{CO}$ Emission}

In the nuclei of type 1 Seyfert galaxies, a small (subparsecscale) region of very dense $\left(\sim 10^{10} \mathrm{~cm}^{-3}\right)$ gas with a velocity dispersion of several thousand kilometers per second exists (the broad line region [BLR]). Recent spectropolarimetric observations (Antonucci and Miller 1985; Miller 1988) demonstrate that a BLR is present in some (all?) type 2 Seyferts but is heavily obscured at optical wavelengths. To date, no molecules have been detected in the BLR, although they may exist if the emitting clouds in the BLR have high enough column densities. Moreover, in both type 1 and type 2 Seyfert galaxies, the emission-line profiles from the $10^{2}-10^{3}$ parsec-scale NLR have broad, blue-asymmetric wings with a typical kinematic extent $\gtrsim 10^{3} \mathrm{~km} \mathrm{~s}^{-1}$ (e.g., Heckman, Miley, and Green 1984).

As noted in $\S$ II, during our observing run of 1986 October, we were able to search for very broad $\left(>10^{3} \mathrm{~km} \mathrm{~s}^{-1}\right) \mathrm{CO}$ emission, which might be a counterpart to either the BLR or the broad wings on the NLR emission lines. This was made possible by the rapid beam-switching technique by which the $1^{\prime}$ 
TABLE 7

CO AND Other LiNe Widths in SEyfert GalaXies

\begin{tabular}{|c|c|c|c|c|c|}
\hline $\begin{array}{l}\text { Name } \\
\text { (1) }\end{array}$ & $\begin{array}{l}W_{\mathrm{co}} \\
(2)\end{array}$ & $\begin{array}{c}W_{\mathrm{HI}} \\
(3)\end{array}$ & $\begin{array}{c}W_{\mathrm{O} \text { III }} \\
\text { (4) }\end{array}$ & $\begin{array}{c}W_{\text {stars }} \\
\text { (5) }\end{array}$ & $\begin{array}{c}\sin i \\
(6)\end{array}$ \\
\hline Mrk 938 & 370 & 490 & 330 & $\cdots$ & \\
\hline NGC 931 & 500 & 450 & 330 & & 0.96 \\
\hline NGC 1068. & 310 & 290 & 1150 & 340 & 0.52 \\
\hline NGC $1365 \ldots$ & 210 & 380 & $\$ 300$ & $\ldots$ & 0.83 \\
\hline NGC 1667. & 460 & 330 & 290 & $\ldots$ & 0.58 \\
\hline MCG 8-11-11 & 400 & 310 & 730 & $\ldots$ & 0.45 \\
\hline NGC $2273 \ldots \ldots \ldots \ldots$ & 280 & 350 & & $\ldots$ & 0.69 \\
\hline Mrk $10 \ldots$ & 580 & 560 & 370 & $\ldots$ & 0.91 \\
\hline NGC 2992 & 350 & 350 & 220 & & 0.94 \\
\hline NGC 3227 & 340 & 390 & 510 & 240 & 0.71 \\
\hline NGC $4051 .$. & 180 & 240 & 250 & 180 & 0.61 \\
\hline NGC $4388 \ldots \ldots \ldots \ldots$ & 280 & 310 & 220 & $\ldots$ & 0.96 \\
\hline Mrk 231 & 370 & (170) & & & \\
\hline NGC 5033 & 460 & 430 & 300 & 330 & 0.84 \\
\hline NGC $5135 \ldots$ & 90 & & 165 & & 0.90 \\
\hline M51 ......... & 130 & 230 & 180 & 170 & 0.71 \\
\hline NGC 5256 . & 470 & $\ldots$ & 390 & $\ldots$ & $\ldots$ \\
\hline Mrk 273 . & 450 & & 700 & $\ldots$ & \\
\hline NGC 5347 . & 140 & 100 & & & 0.56 \\
\hline NGC 5506. & 300 & 320 & 320 & 270 & 0.93 \\
\hline Arp 220 & 500 & $(350)$ & 480 & $\ldots$ & \\
\hline (NGC 6764) & 240 & 250 & 400 & & 0.80 \\
\hline NGC 6814. & 70 & 80 & 130 & 310 & 0.30 \\
\hline IC $5135 \ldots$ & 100 & $\ldots$ & 430 & $\ldots$ & 0.41 \\
\hline NGC 7172 & 600 & & & $\ldots$ & 0.80 \\
\hline NGC 7469 & 360 & 280 & 350 & $\ldots$ & 0.67 \\
\hline NGC 7674 & 190 & 340 & 490 & $\ldots$ & 0.30 \\
\hline
\end{tabular}

Key to cols. (2)-(6) follows:

Col. (2). $-W_{\text {co }}$ : FWHM of the CO 1-0 emission line in $\mathrm{km} \mathrm{s}^{-1}$. Sources for the line widths are listed in col. (5) of Table 2. When more than one observation was available, the quoted $W_{\mathrm{CO}}$ is an average value.

Col. (3). $-W_{\mathrm{HI}}: F W H M$ of $\lambda-21 \mathrm{~cm} \mathrm{H} \mathrm{I} \mathrm{emission} \mathrm{line} \mathrm{in} \mathrm{km} \mathrm{s}^{-1}$ (except for Mrk 231 and Arp 220 where the $\lambda-21 \mathrm{~cm}$ line is in absorption). Sources are Heckman, Balick, and Sullivan 1978, Mirabel and Wilson 1984, and Huchtmeier et al. 1983, and references listed therein. When more than one observation was available, the quoted $W_{\mathrm{H}}$ is an average value.

Col. (4). $-W_{\mathrm{OIII}}: F W H M$ of the $[\mathrm{O}$ III] $\lambda 5007$ nuclear emission line in $\mathrm{km} \mathrm{s}^{-1}$. Sources are Heckman, Miley, and Green 1984, Whittle 1985, Vrtilek and Carleton 1985, Phillips, Charles, and Baldwin 1983, Feldman et al. 1982, Heckman et al. 1981, Edmunds and Pagel 1982, and unpublished data of Heckman and Wilson. The quoted $W_{\mathrm{O} \text { III }}$ is an average value when more than one observation is available.

Col. (5).- $W_{\text {stars }}$ : this is defined to be 2.35 times the stellar velocity dispersion $(\sigma)$ in the nucleus, assuming a Gaussian line profile. Sources are Whitmore, McElroy, and Tonry 1986, and references therein and unpublished data of Heckman, Illingworth, and Wilson.

Col. (6).- The sine of the galaxy inclination, computed under the assumption that each galaxy is axisymmetric and infinitesimally thin [i.e., $\sin i \equiv\left(1-R^{-2}\right)^{1 / 2}$, where $R$ is the ratio of major-to-minor axes]. Five galaxies are so peculiar in their optical morphology that no estimation of $\sin i$ was attempted.

beam was chopped against positions $6^{\prime}$ in azimuth from the central position at a rate of $5 \mathrm{~Hz}$. If there were $\mathrm{CO}$ emission with broad-line velocities, it would have appeared as a baseline with a positive offset from zero intensity. We made no detections; had one been made, it would have been necessary to verify that the emission was line emission from the nucleus.

Table 9 summarizes the results for the six galaxies for which we have good data. All but one (NGC 7469) are type 2 Seyferts without an optically prominent BLR. In these cases we are searching for a $\mathrm{CO}$ counterpart to the broad wings on the NLR emission-line profiles, or for CO emission from a BLR that is heavily obscured in the optical. The upper limits to the broad $\mathrm{CO}$ emission was determined as follows. First, the noise in the integrated spectrum $\Delta I(\mathrm{rms})$ is calculated from
$\Delta I(\mathrm{rms})=\Delta T(\mathrm{rms})(\Delta V)\left(N^{1 / 2}\right)$, where $\Delta T(\mathrm{rms})$ is the rms noise per channel, $\Delta V$ is the velocity width of a channel, and $N$ is the number of channels. For galaxies with detected narrow $\mathrm{CO}$ emission lines, $I(\mathrm{CO})$, the integrated emission in the line was determined from the spectrum after removing a baseline from channels with no signal. This was then compared to the emission, integrated over the full bandwidth of the spectrometer with no baseline removed. In all cases the latter emission was within $\sim 2 \Delta I(\mathrm{rms})$ of the value of $I(\mathrm{CO})$. The limits in Table 9 are the values of $\Delta I(\mathrm{rms})$. For NGC 5929 and NGC 1358, we simply compared the emission integrated over the bandwidth with no baseline removed to the value of $\Delta I(\mathrm{rms})$ and found no signal in excess of $\sim 2 \Delta I(\mathrm{rms})$.

The third column in Table 9 gives the ratio of $3 \Delta I(\mathrm{rms})$ to $I(\mathrm{CO})$, the narrow-line $\mathrm{CO}$ emission. In two cases, the ratio is less than half the detected emission of the narrow-line $\mathrm{CO}$. Thus we conclude that in the six Seyferts we observed, any high-velocity CO (in the BLR or NLR) must be a small fraction of the narrow-line $\mathrm{CO}$ emission from the galaxies.

\section{SUMMARY AND IMPLICATIONS}

\section{a) Principal Conclusions}

Our most important conclusion is that type 2 Seyfert galaxies differ systematically from normal disk galaxies and type 1 Seyfert galaxies in the strength of both their millimeter-wave $\mathrm{CO}$ and far-infrared continuum emission. Specifically, we conclude the following:

1. Type 2 Seyferts in the Revised Shapley-Ames Catalog (RSA) have an average ratio of $L_{\mathrm{CO}} / L_{B}$ that is 2-3 times as large as the average ratios for normal disk galaxies and for type 1 Seyferts in the RSA.

2. Type 2 Seyferts in the RSA have an average ratio of $L_{\mathrm{CO}} / M_{\mathrm{HI}}$ that is $\sim 5$ times larger than the average ratio for normal disk galaxies.

3. The type 2 Seyferts in the RSA have an average farinfrared $(\lambda=40-120 \mu \mathrm{m})$ luminosity that is 3 to 4 times larger than the average luminosity of either type 1 Seyfert galaxies in the RSA or normal galaxies having the same Hubble type and absolute magnitude.

4. Dahari and DeRobertis (1988) find that the narrow line region (size scale $0.1-1 \mathrm{kpc}$ ) is significantly more heavily reddened in type 2 than in type 1 Seyferts, suggesting excess dust and cold gas in the type 2 Seyferts.

It has been previously established for galaxies in general that strong $\mathrm{CO}$ emission correlates with both strong nonthermal radio emission and far-infrared thermal emission. Strong CO emission also appears to be well-correlated with a high rate of star formation. For the Seyfert galaxies we find the following:

1. The Seyferts (both types 1 and 2) follow the same correlation between $\mathrm{CO}$ and far-infrared luminosity as normal galaxies and far-infrared-bright non-Seyfert galaxies.

2. While the Seyferts do exhibit a statistically significant correlation between CO luminosity and nonthermal radio power, they are systematically stronger radio sources than non-Seyferts with the same CO luminosity. The Seyferts with the greatest excess radio emission have radio sources whose morphology strongly suggests that they are fueled by a compact active nucleus. 

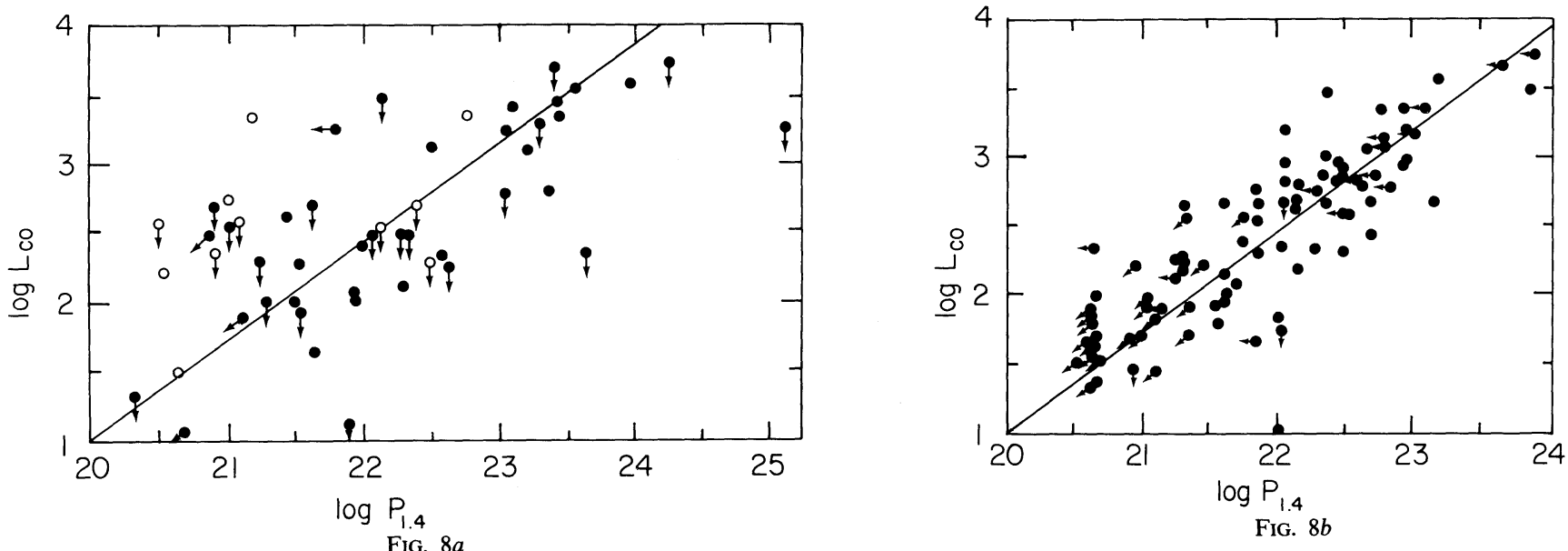

FIG $8-(a)$ Plot of the $\log$ of the $\mathrm{CO}$ luminosity (in $\mathrm{K} \mathrm{km} \mathrm{s}^{-1} \mathrm{kpc}^{2}$ ) vs. the monochromatic radio power at $1.4 \mathrm{GHz}$ (W Hz $\mathrm{Hz}^{-1}$ ) for the Seyfert galaxies in Table 2 . We have used (whenever possible) $1.4 \mathrm{GHz}$ continuum measurements made with low angular resolution to be certain that we have included all the radio flux from the We have Such data are indicated by solid dots. Open dots are Seyfert galaxies for which only VLA A-configuration (high-resolution) data exist. In these cases, the 1.4 Gro hian line is the estimated linear relation between $\log L_{\mathrm{CO}}$ and $\log P_{14}$ for the non-Seyfert galaxies plotted in Fig. $7 b$. This linear regression was calculated using diagonal line is the estimated lo variables. (b) As for $(a)$ but for a sample of Schmitt's (1985) two-dimensional Kaplan-Meier estimator technique which properly treats upper limits in both variables. (b) As for $(a)$ but for a sample of non-Seyfert galaxies. These galaxies were taken from the CO surveys of Stark et al. (1986) and Stark, Elmegreen, and Chance (1987) for normal galaxies, and from Sanders and Mirabel $(1985)$, Sanders et al $(1986)$, and Young et al. $(1984,1986)$ for infrared-bright non-Seyferts. All the radio continuum data were low angular resolution and should represent the total radio power for each galaxy. The diagonal line is the Kaplan-Meier estimator of the linear regression between the two variables.

3. The Seyfert galaxies with the largest CO luminosities show a variety of telltale signs (in the optical, infrared, and radio) of a high rate of star formation.

We have also explored a relationship of the $\mathrm{CO}$ kinematics to the kinematics of the ionized gas in the NLR, the $\mathrm{H}$ I in galaxy disk, and the stars in the central bulge. We have concluded that the $\mathrm{CO}$ is probably arrayed in a rotating disk that is coplanar with the $\mathrm{HI}$ /stellar disk. In particular, we find the following:

1. The CO line widths correlate well with the sine of the estimated galaxy inclination.

2. The $\mathrm{CO}$ and $\mathrm{H}$ I line widths are well-correlated (both the measured widths, and the widths corrected for inclination).

3. The line widths in the NLR (uncorrected for galaxy inclination) correlate much better with the $\mathrm{CO}$ line widths when the latter have been corrected for inclination. Moreover, the ratio of CO-to-NLR line widths is well correlated with the sine of the galaxy inclination.
4. The NLR line widths correlate better with the CO line widths than with the $\mathrm{H}$ I line widths.

5. The CO line widths apparently correlate with the stellar velocity dispersion, and the correlation improves when the $\mathrm{CO}$ line widths are corrected for inclination. However, the confirmation of these results requires a larger sample of Seyfert galaxies with measured stellar velocity dispersions.

6. We find no CO counterparts to either the BLR or the broad (greater than $1000 \mathrm{~km} \mathrm{~s}^{-1}$ ) wings seen on the profiles of the NLR emission lines.

\section{b) Implications}

The above conclusions have some implications for a number of issues concerning Seyfert galaxies.

\section{i) The Structure and Dynamics of the Narrow Line Region}

The $\mathrm{CO}$ versus [O III] line width correlation has two interesting implications for the structure and dynamics of the NLR. First, insofar as the molecular gas is probably gravitationally

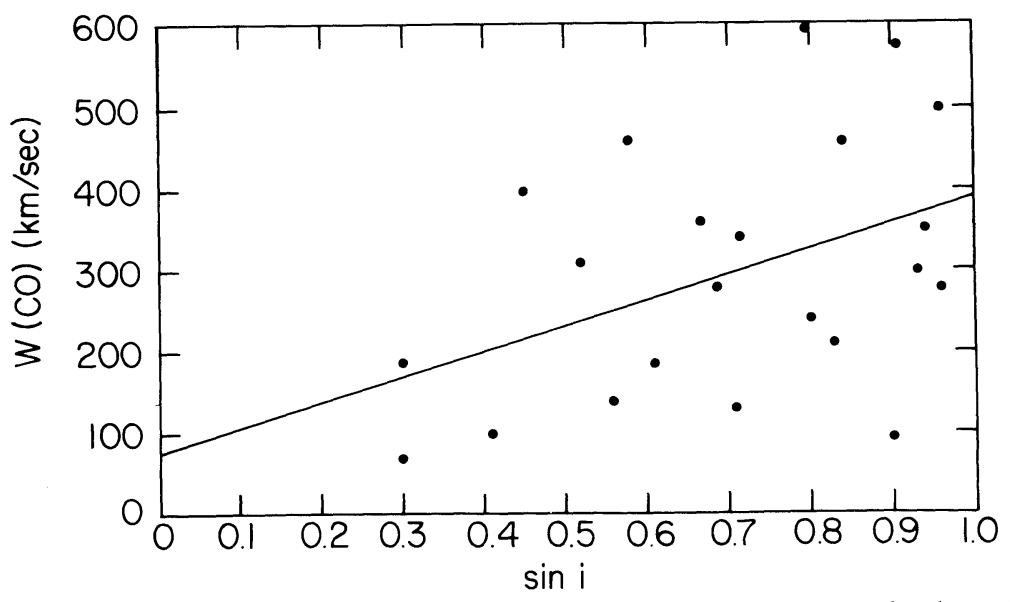

Fig. 9. - The CO line width $\left(\mathrm{km} \mathrm{s}^{-1}\right)$ is plotted against the sine of the galaxy inclination (see Table 7 and associated notes). Solid line shows a least-squares fit to the data. The CO line width and $\sin i$ are correlated at the $97.5 \%$ confidence level (see Table 8 ). 
TABLE 8

LiNE WidTH CORRELATIONS

\begin{tabular}{|c|c|c|c|c|c|c|c|}
\hline $\begin{array}{l}\text { Equation } \\
\text { (1) }\end{array}$ & $\begin{array}{c}a \\
(2)\end{array}$ & $\begin{array}{c}b \\
(3)\end{array}$ & $\begin{array}{c}r \\
(4)\end{array}$ & $\begin{array}{c}n \\
(5)\end{array}$ & $\begin{array}{c}t \\
(6)\end{array}$ & $\begin{array}{l}\text { Significance } \\
\text { (7) }\end{array}$ & $\begin{array}{l}\text { Figure } \\
\text { (8) }\end{array}$ \\
\hline$W_{\mathrm{CO}}=a \sin i+b \ldots \ldots \ldots$ & 313 & 80 & 0.42 & 22 & 2.09 & $97.5 \%$ & 10 \\
\hline$W_{\mathrm{CO}}=a W_{\mathrm{HI}}+b \ldots \ldots \ldots$ & 0.84 & 52 & 0.72 & 22 & 4.65 & $>99.5 \%$ & $11 a$ \\
\hline$\frac{W_{\mathrm{CO}}}{\sin i}=a \frac{W_{\mathrm{HI}}}{\sin i}+b \ldots \ldots \ldots$ & 0.68 & 128 & 0.70 & 19 & 4.04 & $>99.5 \%$ & $11 b$ \\
\hline$W_{\mathrm{Co}}=a W_{\mathrm{O} \text { III }}+b \ldots \ldots \ldots$ & 0.18 & 244 & 0.28 & 23 & 1.29 & $90 \%$ & $12 a$ \\
\hline$W_{\mathrm{HI}}=a W_{\mathrm{O} \text { III }}+b \ldots \ldots \ldots$ & 0.054 & 308 & 0.12 & 19 & 0.47 & $67 \%$ & $\ldots$ \\
\hline$\frac{W_{\mathrm{CO}}}{\sin i}=a W_{\mathrm{O} \text { III }}+b \ldots \ldots \ldots$ & 0.50 & 246 & 0.54 & 19 & 2.66 & $99 \%$ & $12 b$ \\
\hline$\frac{W_{\mathrm{HI}}}{\sin i}=a W_{\mathrm{O} \text { III }}+b \ldots \ldots \ldots$ & 0.37 & 346 & 0.44 & 17 & 1.89 & $97 \%$ & $\ldots$ \\
\hline$\frac{W_{\mathrm{CO}}}{U}=a \sin i+b \ldots \ldots \ldots$ & 1.34 & -0.05 & 0.63 & 19 & 3.35 & $>99.5 \%$ & 13 \\
\hline$W_{\mathrm{CO}}=a W_{*}+b \ldots \ldots \ldots \ldots$ & 0.89 & 23 & 0.45 & 7 & 1.14 & $85 \%$ & $14 a$ \\
\hline$\frac{W_{\mathrm{Co}}}{\sin i}=a W_{*}+b \ldots \ldots \ldots \ldots$ & 1.52 & -20 & 0.65 & 7 & 1.52 & $94 \%$ & $14 b$ \\
\hline
\end{tabular}

Key to cols. (1) $-(8)$ follows:

Col. (1).-Equation representing the least-squares fit to the data in Table 7 and displayed in the corresponding figure.

Cols. (2), (3).- Least-squares fit to the values for $a$ and $b$ in the equation in col. (1)

Col. (4).-Correlation coefficient of the relationship.

Col. (5).--Number of data points in the sample.

Col. (6). - The value of the Student's $t$-statistic for the correlation

$$
t \equiv \frac{r(n-2)^{1 / 2}}{\left(1-r^{2}\right)^{1 / 2}} \text {. }
$$

Col. (7).-Statistical significance of the correlation implied by the value for $t$ in col. (6) for $n-2$ degrees of freedom.

Col. (8).-Figure illustrating the relationship.

bound to the galaxy, it represents additional evidence that gravity plays an important role in the dynamics of the NLR (see also Wilson and Heckman's 1985 review and Whittle 1988). Second, Figure 12 implies that the primary structure of the NLR is not that of a rotating disk that is coplanar with the galaxy disk. This reinforces the conclusions drawn on the basis of the lack of any correlation between [O III] line width and galaxy inclination in large samples of Seyferts (see Heckman et al. 1981; Whittle 1985). Among the alternatives for the NLR, a

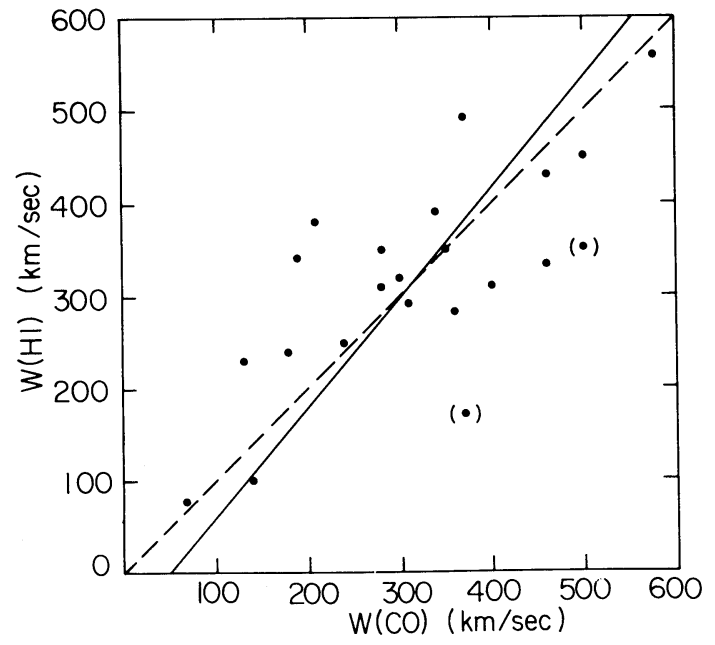

Fig. $10 a$ quasi-spherical infall model, or a hybrid model involving highspeed outflow plus a gravitationally bound component remain viable.

ii) “Fueling” of Nuclear Activity

It is tempting to interpret the systematically strong $\mathrm{CO}$ emission of the type 2 Seyfert galaxies (relative to normal spiral galaxies) as evidence that the Seyfert phenomenon is fueled by molecular gas. Before this interpretation can be taken seriously, a number of points need to be clarified.

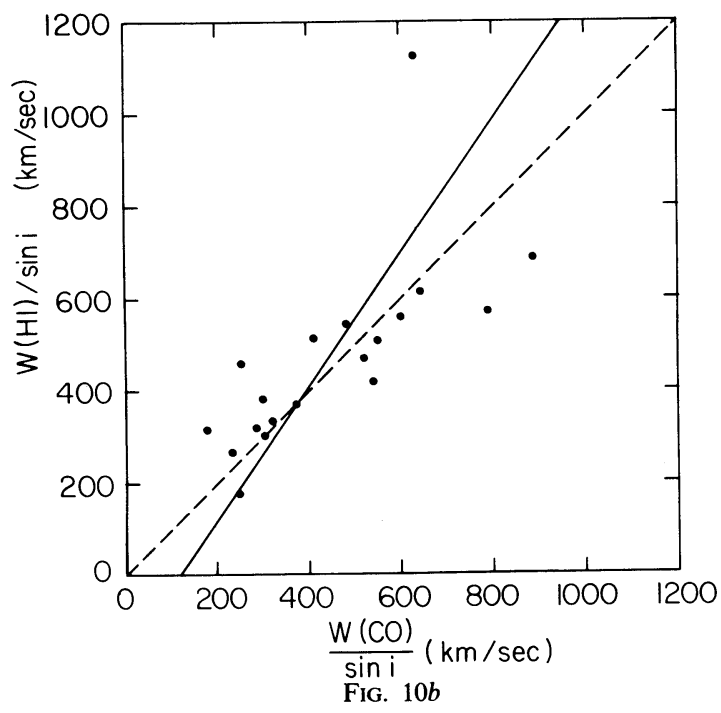

FIG. 10.-The $\mathrm{CO}$ and $\mathrm{H}_{\mathrm{I}} \lambda-21 \mathrm{~cm}$ line widths are plotted against one another. Least-square fits to the data are indicated by the solid lines, while the dashed lines

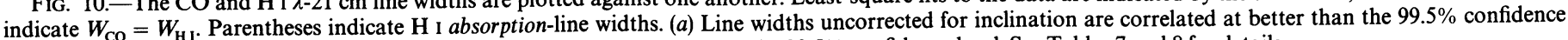
level. (b) Line widths corrected for inclination are also correlated at better than the $99.5 \%$ confidence level. See Tables 7 and 8 for details. 
TABLE 9

LIMITS TO BROAD-LINE CO EMISSION

\begin{tabular}{cccc}
\hline \hline $\begin{array}{c}\text { Galaxy } \\
(1)\end{array}$ & $\begin{array}{c}\text { Seyfert Type } \\
(2)\end{array}$ & $\begin{array}{c}\Delta I(\mathrm{rms}) \\
(3)\end{array}$ & $\begin{array}{c}3 \Delta I(\mathrm{rms}) / I(\mathrm{CO}) \\
(4)\end{array}$ \\
\hline NGC $1358 \ldots \ldots \ldots$ & 2 & 1.0 & $\ldots$ \\
NGC $5135 \ldots \ldots \ldots \ldots$ & 2 & 2.6 & 1.0 \\
NGC $5347 \ldots \ldots \ldots \ldots$ & 2 & 0.4 & 0.4 \\
NGC $5929 \ldots \ldots \ldots$. & 2 & 1.3 & $\ldots$ \\
NGC $7172 \ldots \ldots \ldots \ldots$ & 2 & 1.4 & 0.9 \\
NGC $7469 \ldots \ldots \ldots$ & 1 & 1.2 & 0.3 \\
\hline
\end{tabular}

Key to cols. (3)-(4) follows:

Col. (3).- The expected rms noise in the integrated spectrum:

$$
\Delta I(\mathrm{rms}) \equiv \Delta T(\mathrm{rms}) \Delta v N^{1 / 2} \mathrm{~K} \mathrm{~km} \mathrm{~s}^{-1},
$$

where $\Delta T(\mathrm{rms})$ is the channel noise $(\mathrm{K}), \Delta v$ is the channel width $(\mathrm{km}$ $\left.\mathrm{s}^{-1}\right)$, and $N$ is the total number of channels. We regard $3 \Delta I(\mathrm{rms})$ as a conservative upper limit to the total flux from "broad-line" $\mathrm{CO}$ emis$\operatorname{sion}\left(\Delta v \gtrsim 1000 \mathrm{~km} \mathrm{~s}^{-1}\right)$.

Col. (4). $-3 \Delta I(\mathrm{rms})$ relative to the detected "narrow-line" $\mathrm{CO}$ flux from Table 1.
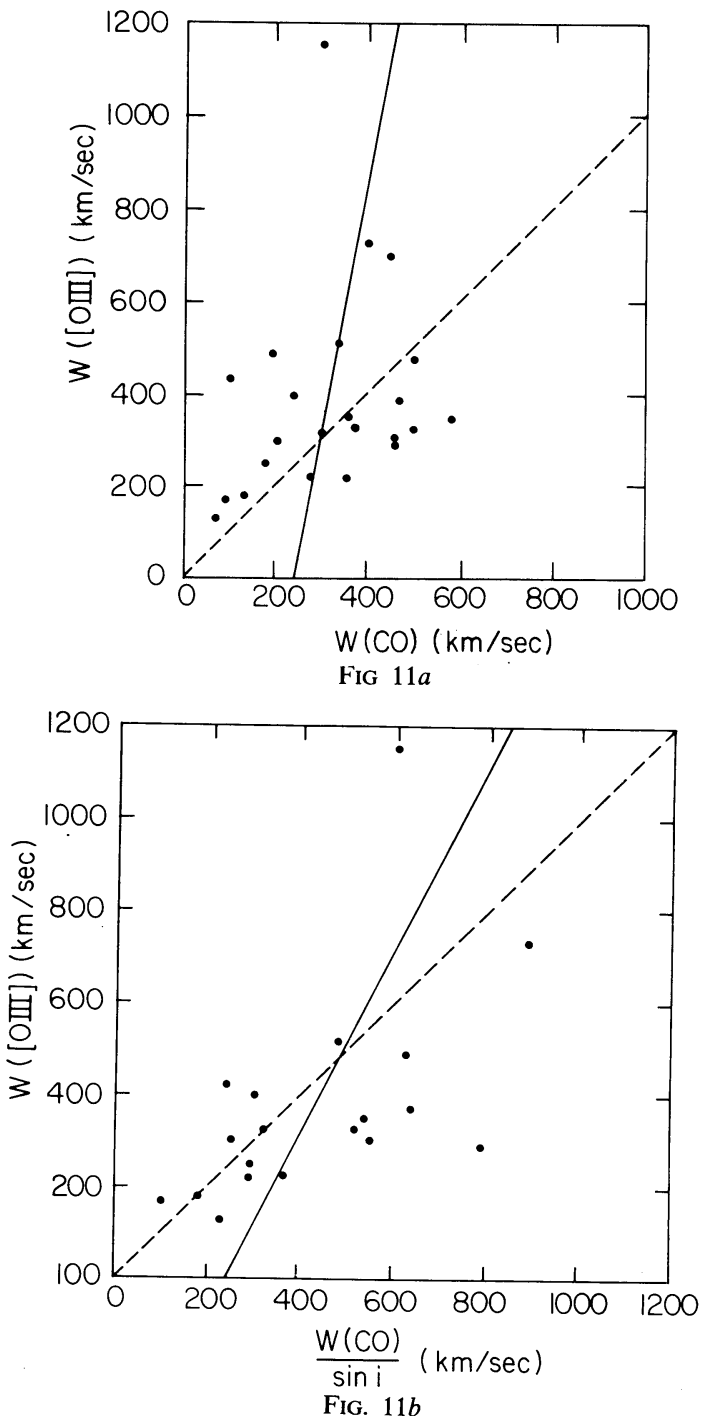

FIG. 11.-Plots of the $\mathrm{CO}$ line widths against the line widths of the nuclear [O III] $\lambda 5007$ emission line. In each figure, solid line is a least-squares fit while the dashed line indicates $W_{\mathrm{CO}}=W_{\mathrm{rO} \mathrm{II}]}$. $(a)$ The line widths with no inclination correction are correlated at only the $90 \%$ confidence level (not significant). (b) The CO line width (corrected for inclination) is plotted against the [O III] line width (with no inclination correction). These parameters are correlated at the 99\% confidence level (see Tables 7 and 8 for details).
First, does the high CO luminosity correspond to a greater molecular hydrogen mass in the type 2 Seyferts, or simply to greater emissivity in the ${ }^{12} \mathrm{CO} J=1-0$ line? The proper procedure for the conversion of $\mathrm{CO}$ luminosities to $\mathrm{H}_{2}$ masses remains a controversial topic in extragalactic astronomy. The "standard" conversion factor (Bloemen et al. 1986; Israel and Rowan-Robinson 1984; Sanders, Solomon, and Scoville 1984) appears to be reliable to better than a factor of a few when applied to Giant Molecular Clouds (GMCs) in the disk of the Milky Way (e.g., Dame et al. 1987). However, the physical state of the molecular gas in active galaxies may be quite different from that in normal GMCs leading to a significantly different ${ }^{12} \mathrm{CO} J=1$ emissivity per unit $\mathrm{H}_{2}$ mass. Indeed, several lines of evidence suggest that the standard $L_{\mathrm{CO}}-$ to $-M_{\mathrm{H}_{2}}$ conversion factor may overestimate $M_{\mathrm{H}_{2}}$ in at least some galactic nuclei (see the recent review by Blitz 1988 and papers by Sofue 1988; Rickard and Blitz 1985; Blitz et al. 1985; Lo et al. 1987). Detailed observations of other CO transitions (e.g., the more optically thin ${ }^{13} \mathrm{CO} J=1-0$ line and the higher excitation ${ }^{12} \mathrm{CO} J=2-1$ and $J=3-2$ lines) would be useful as probes of the physical conditions in the molecular gas in Seyfert galaxies.

Second, establishing a clear causal connection between molecular gas and the fueling of nuclear activity will require high-resolution maps of the CO emission in Seyferts. These will determine whether the molecular gas has an unusually high degree of central concentration and/or significant noncircular motions.

Finally, the apparently normal CO properties of the type 1 Seyfert galaxies must be understood. If Seyfert activity is fueled by an excess of circumnuclear molecular gas, why do the type 1 Seyfert galaxies show no sign of such an excess? We will return to this issue below when we discuss the relationship of the Seyfert types and present a possible evolutionary scenario for active galaxies.

\section{iii) The Origin of the Far-Infrared Continuum in Seyfert Galaxies}

As noted in $\S I$, the nature of the far-infrared emission in Seyfert galaxies is still not well understood. In particular, the relative importance of nonthermal emission, thermal reradiation of nuclear nonthermal emission, and thermal reradiation of starlight may vary between the two Seyfert types and as a function of the luminosity of the active nucleus. The $\mathrm{CO}$ data offer an important new perspective. The fact that Seyfert galaxies follow the same correlation between $\mathrm{CO}$ and far-infrared luminosities as normal and far-infrared-bright non-Seyfert galaxies suggests that (for the set of Seyfert galaxies in Table 2 at least), the bulk of the far-infrared emission is likely to be reradiated starlight.

We add the parenthetical remark above because Table 2 is biased in favor of Seyfert galaxies that are either bright in the optical (and are hence relatively nearby galaxies with usually rather modest nuclear luminosities) or bright in the farinfrared. Many of the Markarian or Zwicky-compact type 1 Seyfert galaxies (i.e., galaxies that have nuclei that are very luminous in the optical and ultraviolet, but that exhibit rather weak far-infrared excesses) might not follow the $L_{\mathrm{CO}}$ versus $L_{\text {FIR }}$ relationship, since they could well have a strong nonthermal FIR component (Edelson, Malkan, and Rieke 1987). This is even more likely to be the case in most highly luminous quasars (e.g., Neugebauer et al. 1986).

iv) The Origin of Nonthermal Radio Emission in Seyfert Galaxies

We believe that the most plausible interpretation of the results presented above is that the radio continuum radiation 


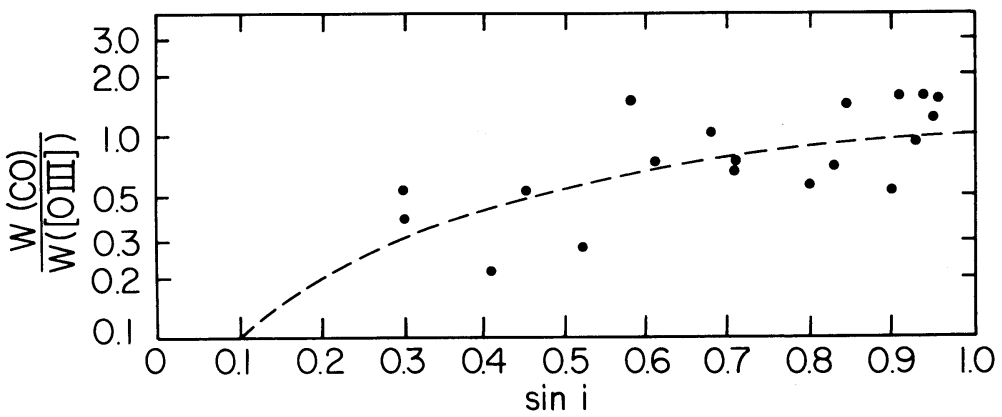

FIG. 12.-The ratio of the $\mathrm{CO}-$ to- $[\mathrm{O}$ III] line widths plotted against the sine of the estimated galaxy inclination (see Table 7). The correlation between the two parameters is significant at better than the $99.5 \%$ confidence level (see Table 8). The curved dashed line indicates the relation $W([\mathrm{O} \mathrm{III}])=\mathrm{W}(\mathrm{CO}) / \mathrm{sin} i$.

of Seyfert galaxies has two components-one fueled by star formation, perhaps in the form of multiple supernova remnants, and the other by nonthermal nuclear activity. The first component is presumably closely related to the molecular gas content and is responsible for the broad correlation in Figure $8 a$, while the second may be independent of $\mathrm{CO}$ and shows up in galaxies with greater radio emission than expected from the relation. Such a composite nature for the radio emission is known to apply to individual well-studied galaxies, such as NGC 1068 (Wilson and Ulvestad 1982; Wynn-Williams, Becklin, and Scoville 1985) and NGC 7469 (Ulvestad, Wilson, and Sramek 1981; Condon et al. 1982). As noted earlier, the signature of star formation in the radio continuum is a morphology which is not jetlike or double. Sources with this type of "diffuse" morphology are found in galaxies with prominent, ongoing star formation as evidenced by $\mathrm{H}$ II regions, either circumnuclear or in the disk (Wilson 1987). Our CO study strongly supports the view that the component of radio continuum emission in Seyferts that is not directly related to nuclear activity is fueled by ongoing star formation processes.

v) The Relationship of Nuclear Activity to Star Formation

The strength of the far-infrared emission, the correspond- ingly strong $\mathrm{CO}$ emission, and the optical, radio, and infrared evidence summarized above all imply that type 2 Seyfert galaxies are probably characterized by a higher than normal rate of star formation. While this suggests that there is a causal relationship between star formation and Seyfert activity, the nature of this relationship cannot be delineated from the present data alone. It is particularly perplexing that the type 1 Seyfert galaxies show no evidence for an unusually large star formation rate. As we will suggest below, this may be an evolutionary effect.

vi) The Relationship between Type 1 and Type 2 Seyfert Galaxies

This has proved to be one of the most durable of the problems associated with the Seyfert phenomenon. A growing consensus (e.g., Antonucci and Miller 1985; Miller 1988) holds that type 2 Seyferts are simply type 1 Seyferts in which the observer's view of the centralmost region $(\lesssim \mathrm{pc})$ is heavily obscured (at least at optical, ultraviolet, and X-ray wavelengths). The most extreme form of this hypothesis would be that there are no intrinsic differences between the two types and that whether a Seyfert galaxy is classified as type 1 or 2 depends solely on the direction from which it is viewed. This hypothesis can be convincingly ruled out by our results on the striking differences in

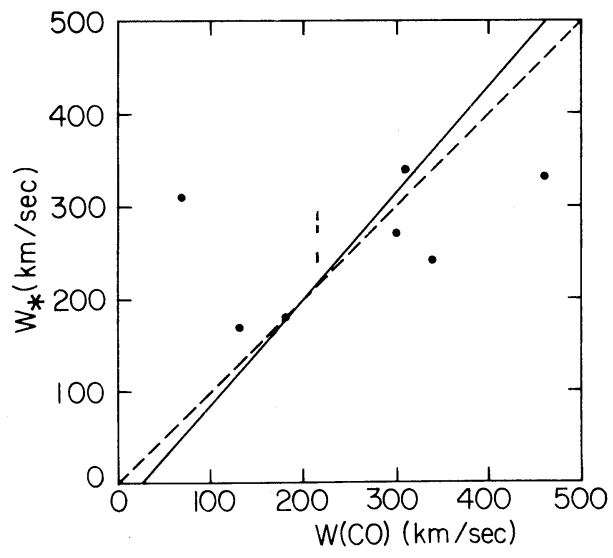

Fig. $13 a$

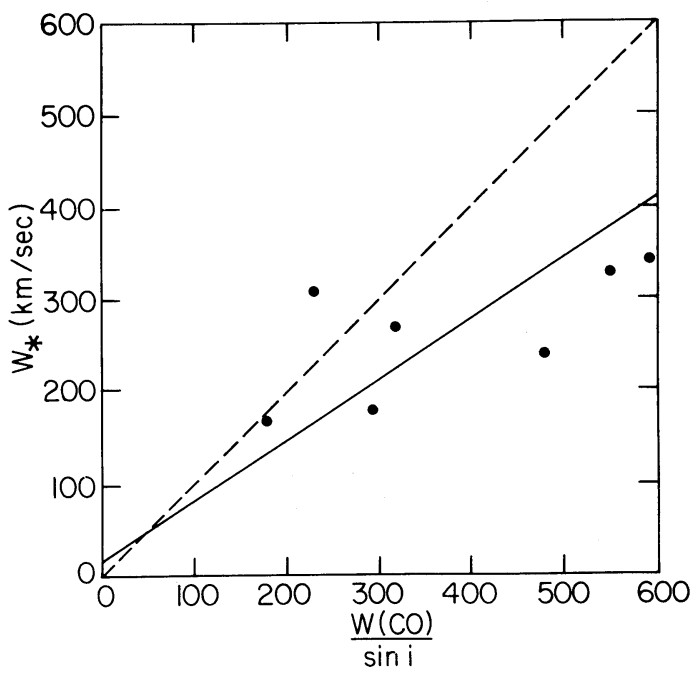

Fig. $13 b$

FIG. 13.-Plots of the CO line width vs. the width of the nuclear stellar absorption lines $\left(W_{*} \equiv 2.35 \sigma\right.$, where $\sigma$ is the usual stellar velocity dispersion). Solid lines indicate least-square fits, while dashed lines indicate $W_{\mathrm{CO}}=W_{*} .(a)$ Line widths with no inclination correction are correlated at only the $84 \%$ confidence level. $(b)$ The CO line width corrected for inclination is correlated with the uncorrected $W_{*}$ at the $94 \%$ confidence level (marginally significant). A larger sample is required to confirm this result. See Tables 7 and 8 for details. 
the viewing-angle-independent $\mathrm{CO}$ and far-infrared properties between the two Seyfert types.

A more plausible form of the above hypothesis is that Seyfert galaxies are characterized by a range in the nuclear "covering fraction" (fraction of the sky, as seen from the nucleus, that is covered by the obscuring medium). The difference between type 1 and 2 Seyferts would then be due to both intrinsic differences and viewing-angle effects. That is, Seyfert 2's have larger average covering fractions, but some objects classified as type 1 (type 2) Seyferts are objects with large (small) covering fractions viewed from "lucky" ("unlucky") directions.

While this picture has gained increasing popularity, the nature of the putative obscuring medium is not yet determined. Clearly, it must be large enough to obscure the broad-line region ( $\gtrsim 1 \mathrm{pc}$ ), but small enough to not obscure the narrowline region ( $\lesssim$ few hundred parsecs). Krolik and Begelman $(1986,1988)$ argue that it is most likely a molecular torus created and sustained by the accretion of molecular gas from larger radii. This ties in rather nicely with the excess $\mathrm{CO}$ emission in the type 2 Seyferts (even though most of this emission probably arises on scales well outside the occulting molecular torus itself). Independent of any particular model, it seems plausible to link the apparent excess of molecular gas in type 2 Seyferts to an obscuring medium.

The differences in the $\mathrm{CO}$ and far-infrared properties of the type 1 and 2 Seyfert galaxies can also be used to set a lower limit to the time scale over which the population of Seyferts could have their types transmuted. Since we have argued that the far-infrared emission is powered by dust reradiation of starlight, one such lower limit is just the lifetime of an OB star (few million years). The other lower limit would be the time scale over which molecular clouds (and dust grains) can be created and destroyed. This time scale is very difficult to estimate in the unusual environment in which circumnuclear molecular clouds in an active galaxy are located.

vii) A Possible Evolutionary Scenario for Active Galaxies

As we have emphasized repeatedly, the relationships between star formation and nuclear activity and between type 1 and type 2 Seyfert galaxies may prove central to the phenomenon of the AGN. In what follows, we outline a speculative scenario that attempts to unify the starburst and Seyfert phenomenon. This scenario is an attempt both to synthesize some recent similar ideas in this vein (e.g., Weedman 1983, Sanders et al. 1988; Norman and Scoville 1988; Terlevich and Melnick 1985; Heckman, Armus, and Miley 1987; Rieke, Lebofsky, and Walker 1988) and to include explicitly type 2 Seyfert galaxies as the "missing link" between far-infrared galaxies (FIRGs) and type 1 Seyferts and quasars. We note that powerful radio galaxies could be included (perhaps as a parallel sequence) in the following scenario, particularly in light of recent evidence that many are strong far-infrared sources (Golembek, Miley, and Neugebauer 1987) and often show evidence for recent or on-going star formation (McCarthy 1987; Lilly and Longair 1984; Smith 1988; Smith and Heckman 1987, 1988).

We begin by assuming that some event or process has led to the concentration of a large mass $\left(10^{9}-10^{10} M_{\odot}\right)$ of molecular gas in the circumnuclear (kiloparsec-scale) region of a galaxy. Note that molecular cloud collisions are highly inelastic and are likely to provide for the infall of the molecular material. Galaxy collisions or mergers have been implicated in the genesis of many classes of active galaxy (e.g., Armus, Heckman, and Miley 1987; Dahari 1985; Heckman et al. 1987; Hut- chings, Crampton, and Campbell 1984; Keel et al. 1985; Kennicutt et al. 1987; Sanders et al. 1988), and the physical process by which such events would produce a circumnuclear concentration of gas has been studied with increasing detail (see Toomre and Toomre 1972; Noguchi 1988). Other mechanisms might also be important, however (e.g., bars and/or oval distortions leading to noncircular gas motions, dissipation, and inflow; see Simkin, Su, and Schwarz 1980; Schlosman, Frank, and Begelman 1988).

As discussed by Norman and Scoville (1988), Larson (1988), and others, if self-gravity is important in the circumnuclear molecular disk, dynamical instabilities will quickly lead to a burst of star formation. If a massive black hole is already present in the nucleus, it can be efficiently fueled as well (see Norman 1987), so that an AGN will then accompany the starburst (we note in passing that Norman and Scoville 1988 and Weedman 1983 have proposed instead that the starburst leads directly to the formation of the black hole, or holes). Initially, both the newly formed stars and the AGN will be shrouded in the molecular gas that spawned them. The bulk of the emitted energy will then emerge as reprocessed mid/far-infrared thermal radiation. This is the FIRG stage, characterized by very strong far-infrared and CO emission (e.g., Sanders et al. 1988).

The starburst will impart a significant amount of thermal and mechanical energy to the molecular gas. Supernova explosions, Wolf-Rayet winds, and radiation pressure should suffice to excavate the circumnuclear region gradually (the AGN could also play an important energetic role in this process). The resulting formation of a largescale mass outflow ("superwind") has been recently investigated theoretically by Chevalier and Clegg (1985) and Tomisaka and Ikeuchi (1988) and observationally by Heckman, Armus, and Miley (1987, 1989), McCarthy, Heckman, and van Breugel (1987), Phillips et al. (1983), Sofue (1988), and Bland and Tully (1988). At this stage, the AGN proper remains obscured along most lines of sight and the optical emission-line spectrum is characterized by unusually broad forbidden lines and line ratios that are rarely Seyfert-like (they are instead either of the LINER class or intermediate between LINERs and giant $H$ II regions; Heckman, Armus, and Miley 1987; Armus, Heckman, and Miley 1989). The broad lines arise in the superwind, while the line ratios may reflect the competing processes of shock heating by the superwind and photoionization by the diffuse radiation from the partially excavated starburst and AGN.

As the circumnuclear region gradually emerges from its dust shroud, the galaxy enters the Seyfert 2 stage. It will still be an unusually strong source of far-infrared and millimeter-wave $\mathrm{CO}$ emission, since the starburst is not yet quenched. The centralmost nucleus in which the nonstellar continuum source and broad line region arise are still obscured along most lines of sight, but can be observed in scattered (highly polarized) light that leaks out along favored directions (e.g., along the axis of the radio jets, as suggested by Antonucci and Miller 1985; Wilson, Ward, and Haniff 1988, and others). The optical emission-line spectrum is now dominated by gas photoionized by the AGN (the classical NLR), although circumnuclear giant $\mathrm{H}$ II regions may also be conspicuous. The type 2 Seyfert galaxy NGC 1068 is a prototype for this evolutionary phase (see Baldwin, Wilson, and Whittle 1987; Balick and Heckman 1985; Telesco et al. 1984; Wynn-Williams, Becklin, and Scoville 1985; Scoville, Young, and Lucy 1983). During this phase, if we happen to view the nucleus from a favored (unobscured) 
direction, the BLR and nonstellar optical/X-ray continuum source may be visible. NGC 7469 and Mrk 231 are good examples of such a case, since they are type 1 Seyfert galaxies with very strong $\mathrm{CO}$ and far-infrared emission.

Finally, the circumnuclear molecular reservoir is depleted (by star formation and wind-driven outflow), obscuration is no longer important, and the central nucleus blazes in naked splendor. Depending on the power of the AGN, a type 1 Seyfert galaxy or quasar is born. The galaxy will no longer be a strong $\mathrm{CO}$ source, and any excess far-infrared emission will arise predominantly in the compact $\mathrm{AGN}$ by nonthermal processes. This picture is further supported by the results of Edelson, Malkan, and Rieke (1987) on the much greater relative strength of thermal versus nonthermal infrared emission in Seyfert 2 versus Seyfert 1 galaxies and QSOs. In fact, careful inspection of a sample of type 1 Seyfert galaxies might reveal a deficiency of molecular gas in the central kiloparsec-scale region relative to normal spiral galaxies. Testing this hypothesis must await a program of interferometric mapping of $\mathrm{CO}$ in a large sample of active and normal galaxies (a program some of us have just begun; see Heckman 1989). Once the central engine has used up all the remaining fuel, it will then shut down. The clues that the resulting normal galaxy was recently active may include a central $\mathrm{CO}$ "hole" and a "post starburst" optical and near-infrared spectrum on circumnu- clear scales (see Rieke, Lebofsky, and Walker 1988; Walker, Lebofsky, and Rieke 1988).

This scenario predicts that there might be a systematic difference in the morphology of the host galaxy between FIRGs (highly distorted morphology caused by a very recent or even on-going galaxy interaction), type 2 Seyferts (intermediate evolutionary state with a less distorted morphology), and type 1 Seyferts (advanced evolutionary state with only mild morphological peculiarities). Clearly, FIRGs are generically galaxies with very distorted optical morphologies (e.g., Armus, Heckman, and Miley 1987; Sanders et al. 1988). As emphasized by Blank and Soker (1988), the data of Dahari (1984) imply that type 2 Seyferts might typically be more strongly interacting systems than type 1 Seyferts. These results are at least qualitatively consistent with the evolutionary scenario we have proposed.

We would like to thank the staff at the NRAO $12 \mathrm{~m}$ telescope for their help in obtaining the data presented in this paper. T. M. H. and L. A. are supported by NSF grant AST 85-15896. T. M. H., L. A., and A. S. W. are also supported by NASA/IRAS grants. L. B. is supported by NSF grant AST 86-18763. L. B. thanks the Institute for Advanced Study for its hospitality during the time this paper was being written.
Antonucci, R. R. J., and Miller, J. S. 1985, Ap. J., 297, 621

Armus, L., Heckman, T. M., and Miley, G. K. 1987, A.J., 94, 831.

Armus, L., Heckman,

Baldwin, J., Wilson, A. S., and Whittle, M. 1987, Ap. J., 319, 84.

Balick, B., and Heckman, T. M. 1982, Ann. Rev. Astr. Ap., 20, 431.

. 1985, A.J., 90, 197.

Balzano, V. A. 1983, Ap. J., 268, 602.

Bieging, J., Blitz, L., Lada, C., and Stark, A. 1984, Ap. J., 247, 443

Bland, J., and Tully, J. R. 1988, Nature, in press.

Blank, D. L., and Soker, N. 1988, in Active Galactic Nuclei, ed. H. R. Miller and P. J. Wiita (Berlin: Springer), in press.

Blitz, L. 1988, in Molecular Astrophysics, ed. T. W. Hartquist (Cambridge: Cambridge University Press), in press.

Blitz, L., Bloemen, J., Hermsen, W., and Bania, T. 1985, Astr. Ap., 143, 267

Blitz, L., Mathieu, R. D., and Bally, J. 1986, Ap. J., 311, 142.

Bloemen, J., et al. 1986, Astr. Ap., 154, 25

Carleton, N. P., Elvis, M., Fabbiano, G., Willner, S. P., Lawrence, A., and Ward, M. 1987, Ap. J., 318, 595.

Chevalier, R. A., and Clegg, A. W. 1985, Nature, 317, 44.

Condon, J. J. 1988, preprint.

. 1980, Ap. J., 242, 894.

Condon, J. J., and Broderick, J. 1988, preprint.

Condon, J. J., Condon, M. A., Gisler, G., and Puschell, J. J. 1982, Ap. J., 252, 102.

Dahari, O. 1984, Ph.D. thesis, University of California at Santa Cruz 1985, Ap. J.Suppl., 57, 643.

Dahari, O., and DeRobertis, M. 1988, preprint

Dame, T. M., et al. 1987, Ap. J., 322, 706.

de Vaucouleurs, G., de Vaucouleurs, A., and Corwin, H. 1976, The Second Reference Catalogue of Bright Galaxies (Austin: University of Texas).

Dressel, L. L., and Condon, J. J. 1978, Ap. J. Suppl., 36, 53.

Edelson, R. A. 1987, Ap. J., 313, 651.

Edelson, R. A., and Malkan, M. A. 1986, Ap. J., 308, 59.

Edelson, R. A. Malkan, M. A., and Rieke, G. H. 1987, Ap. J., 321, 233.

Edmunds, M. G., and Pagel, B. E. J. 1982, M.N.R.A.S., 198, 1089.

Elmegreen, D. M., and Elmegreen, B. G. 1982, A.J., 87, 626.

Feigelson, E. D., and Nelson, P. I. 1985, Ap. J., 293, 192.

Feldman, F. R., Weedman, D. W., Balzano, V. A., and Ramsey, L. W. 1982, Ap. $J ., 256,427$.

Fisher, J. R., and Tully, R. B. 1981, Ap. J. Suppl., 47, 139.

Golembek, D., Miley, G. K., and Neugebauer, G. 1988, A.J., 95, 26.

Heckman, T. M. 1988, in Starbursts and Galaxy Evolution, ed. T. Montmerle (Paris: Editions Frontiers), p. 381

1989, in IAU Symposium 134, Active Galactic Nuclei, ed. J. S. Miller and D. E. Osterbrock (Mill Valley, CA: University Science Books), in press.

Heckman, T. M., Armus, L., and Miley, G. K. 1987, A.J., 93, 276.

\section{REFERENCES}

Heckman, T. M., Armus, L., and Miley, G. K. 1989, in preparation.

Heckman, T. M., Balick, B., and Sullivan, W. T. 1978, Ap. J., 224, 745

Heckman, T. M., Miley, G. K., Butcher, H. R., and van Breugel, W. 1981, Ap. J., 247, 403 .

Heckman, T. M., Miley, G. K., and Green, R. F. 1984, Ap. J., 281, 525.

Heckman, T. M., Smith, E. P., Baum, S. A., van Breugel, W., Miley, G. K. Illingworth G. D. Bothun, G. D., and Balick, B. 1987, Ap. J., 311, 526.

Holmberg. E. 1975, in Stars and Stellar Systems, Volume 9, Galaxies and the Universe, ed. A. Sandage, M. Sandage, and J. Kristian (Chicago: University of Chicago Press), p. 123.

Huchra, J., and Berg, R. 1988, in preparation.

Huchtmeier, W. K Richter, O.-G., Bohnenstengel, H.-D., and Hauschaldt, M. 1983, A General Catalog of $\mathrm{H}_{\mathrm{I}}$ Observations of External Galaxies (ESO: Garching).

Hummel, E. 1980, Astr. Ap. Suppl., 41, 151

Hutchings, J. B., Crampton, D., and Campbell, B. 1984, Ap. J., 280, 41

Isobe, T. Feigelson, E. D. and Nelson, P. I. 1986, Ap. J., 306, 490.

Israel, F. P., and Rowan-Robinson, M. 1984, Ap. J., 283, 81

Keel, W. C., Kennicutt, R. C., Hummel, E., and van der Hulst, J. M. 1985, A.J., 90, 708.

Kellerman, K. I., Pauliny-Toth, I. I. K., and Williams, P. J. S. 1969, Ap. J., $157,1$.

Kennicutt, R. C., Keel, W. C., van der Hulst, J. M., Hummel, E., and Roettiger, A. 1987, A.J., 93, 1011

Kirkpatrick, H., Wilson, A. S., and Heckman, T. M. 1989, in preparation.

Kojoian, G., Sramek, R. A., Dickinson, D. F., Tovmassian, H., and Purton, C. R. 1976, Ap. J., 203, 323.

Krolik, J. H., and Begelman, M. C. 1986, Ap. J.(Letters), 308, L55.

. 1988, Ap. J., in press.

Larson, R. B. 1988, in Starbursts and Galaxy Evolution, ed. T. Montmerle (Paris: Editions Frontieres), p. 467

Lilly, S. I., and Longair, M.S. 1984, M.N.R.A.S., 211, 833.

Lo, K. Y., Cheung, K. W., Masson, C. R., Phillips, T. G., Scott, S. L., and Woody, D. P. 1987, Ap. J., 312, 57.

Lonsdale, C. J., Helou, G., Good, J. C., and Rice, W. 1985, Cataloged Galaxies and Quasars Observed in the IRAS Survey (Pasadena: JPL).

Malkan, M. A. 1984, Ap. J., 287, 555.

Malkan, M. A., and Filippenko, A. V. 1983, Ap. J., 275, 477

McCarthy, P. C. 1987, Bull. AAS, 19, 1068.

McCarthy, P. C., Heckman, T. M., and van Breugel, W. 1987, A.J., 93, 264.

Meurs, E., and Wilson, A.S. 1984, Astr. Ap., 136, 206.

Miley, G. K., Neugebauer, G., and Soifer, B. T. 1985, Ap. J. (Letters), 293, L11.

Miller, J. S. 1988, in IAU Symposium 134, Active Galactic Nuclei, ed. J. S. Miller and D. E. Osterbrock (Mill Valley, CA: University Science Books), in press.

Mirabel, I. F., and Wilson, A. S. 1984, Ap. J., 277, 92.

Morris, M., and Rickard, L. J. 1982, Ann. Rev. Astr. Ap., 20, 517. 
Neugebauer, G., Miley, G. K., Soifer, B. T., and Clegg, P. E. 1986, Ap. J., 308, 815.

Nilson, P. 1971, Uppsala General Catalogue of Galaxies (Uppsala: Uppsala University).

Noguchi, M. 1988, Astr. Ap., 203, 259.

Norman, C. A. 1987, in Star Formation in Galaxies, ed. C. Persson (Pasadena: Caltech/JPL), p. 395.

Norman, C. A., and Scoville, N. Z. 1988, Ap. J., 332, 124.

Phillips, M. M., Charles, P. A., and Baldwin, J. A. 1983, Ap. J., 266, 485.

Phillips, M. M., Turtle, A. J., Edmunds, M. G., and Pagel, B. E. J. 1983, M.N.R.A.S., 203, 759 .

Rickard, L. J., and Harvey, P. M. 1984, A.J., 89, 1520.

Rickard, L. J., Turner, B. E., and Palmer, P. 1985, A.J., 90, 1175.

Rickard, L. J., and Blitz, L. 1985, Ap. J. (Letters), 292, L57.

Rieke, G. H., and Lebofsky, M. J. 1979, Ann. Rev. Astr. Ap. 17, 477

Rieke, G. H., Lebofsky, M. J., and Walker, C. E. 1988, Ap. J., 325, 679

Rodriguez-Espinosa, J. M., Rudy, R. J., and Jones, B. 1986, Ap. J., 309, 76

. 1987, Ap.J., 312, 555 .

Rowan-Robinson, M. 1988, in Starbursts and Galaxy Evolution, ed. T. Montmerle (Paris: Editions Frontiere).

Sandage, A., and Tammann, G. A. 1981, A Revised Shapley-Ames Catalog of Bright Galaxies (Washington, DC: Carnegie Institution of Washington).

Sanders, R. H., and Bania, T. M. 1976, Ap. J., 204, 341.

Sanders, D. B., and Mirabel, I. F. 1985, Ap. J. (Letters), 298, L31.

Sanders, D. B., Scoville, N. Z., Young, J. S., Soifer, B. T., Schloerb, F. P., Rice, W. L., and Danielson, G. E. 1986, Ap. J. (Letters), 305, L45.

Sanders, D. B., Soifer, B. T., Elias, J. H., Madore, B. F., Mathews, K., Neugebauer, G., and Scoville, N. Z. 1988, $A$ p. J., 325, 74

Sanders, D. B., Solomon, P., and Scoville, N. Z. 1984, Ap. J., 276, 182

Schmitt, J. H. M. M. 1985, Ap. J., 293, 178

Schlosman, I., Frank, J., and Begelman, M. C. 1988, preprint.

Scoville, N. Z., and Young, J. S. 1983, Ap. J., 265, 148.

Scoville, N. Z., Young, J. S., and Lucy, L. B. 1983, Ap. J., 270, 443

Simkin, S. M., Su, H. J., and Schwarz, M. P. 1980, Ap. J., 237, 404

Smith, E. P. 1988, Ph.D. thesis, University of Maryland

Smith, E. P., and Heckman, T. M. 1987, in Radio Continuum Emission in Clusters of Galaxies, ed. J. Uson and C. O'Dea (NRAO: Charlottesville), $\mathrm{p}$ 305 .

.1989, Ap. J., in press.

Smith, E. P., Heckman, T. M., Bothun, G. D., Romanishin, W. R., and Balick, B. 1986, Ap. J., 306, 64.
Sofue, Y. 1988, in Galactic and Extragalactic Star Formation, ed. R. Pudritz and M. Fich (Dordrecht: Reidel), in press.

Stark, A. A., Elmegreen, B. G., and Chance, D. 1987, Ap. J., 322, 64

Stark, A. A., Knapp, G. R., Bally, J., Wilson, R. W., Penzias, A. A., and Rowe, H. E. $1986, A p . J ., 310,660$

Sulentic, J. 1976, Ap. J. Suppl., 32, 171.

Terlevich, R., and Melnick, J. 1985, M.N.R.A.S., 213, 841.

Telesco, C. M., Becklin, E. E., Wynn-Williams, C. G., and Harper, D. A. 1984, Ap. J., 282, 427 .

Tomisaka, K., and Ikeuchi, S. 1988, preprint.

Toomre, A., and Toomre, J. 1972, AP.J., 178, 623.

Tovmassian, H. M. 1966, Australian J. Phys., 19, 883.

Ulvestad, J. S., and Wilson, A. S. 1984, Ap. J., 285, 439

Ulvestad, J. S., Wilson, A. S., and Sramek, R. A. 1981, Ap. J., 247, 419.

Verter, F. 1985, Ap. J. Suppl., 57, 261.

Vrtilek, J. M., and Carleton, N. P. 1985, Ap. J., 294, 106.

Walker, C. E., Lebofsky, M. J., and Rieke, G. H. 1988, Ap. J., 325, 687.

Ward, M., Elvis, M., Fabbiano, G., Carleton, N. P., Willner, S. P., and Lawrence, A. 1987, Ap. J. 315, 74 .

Weedman, D. W. 1983, Ap. J., 266, 479.

Whitmore, B. C., McElroy, D. B., and Tonry, J. L. 1985, Ap. J. Suppl., $59,1$.

Whittle, M. 1985, M.N.R.A.S., 213, 1.

1988, in IAU Symposium 134, Active Galactic Nuclei, ed. J. S. Miller and D. E. Osterbrock (Mill Valley, CA: University Science Books), in press.

Wilson, A. S. 1987, in Star Formation in Galaxies, ed. C. Persson (Pasadena: NASA/JPL), p. 675.

Wilson, A. S., Baldwin, J. A., Sun, S., and Wright, A. E. 1986, Ap. J., 310, 121.

Wilson, A. S., and Heckman, T. M. 1985, in Astrophysics of Active Galaxies and Quasi-Stellar Objects, ed. J. S. Miller (Mill Valley: University Science Books), p. 39.

Wilson, A. S., and Meurs, E. 1982, Astr. Ap. Suppl., 50, 217

Wilson, A. S., and Ulvestad, J. S. 1982, Ap. J., 263, 576

Wynn-Williams, C. G., Becklin, E. E., and Scoville, N. Z. 1985, Ap. J., 297, 607.

Yee, H. K. C. 1983, Ap. J., 272, 473.

Young, J. S., Kenney, J., Lord, S. D., and Schloerb, F. P. 1984, Ap. J. (Letters), 287, L65.

Young, J. S., Scoville, N. Z., and Brady, E. 1985, Ap. J., 288, 487.

Young, J. S., Schloerb, F. P., Kenney, J. D., and Lord, S. D. 1986, Ap. J., 304, 443 .

L. Armus, L. Blitz, T. M. Heckman, and A. S. Wilson: Astronomy Program, University of Maryland, College Park, MD

20742

G. K. MileY: Sterrewacht, Niels Bohringer 2, P.O. Box 9513, 2300 RA Leiden, The Netherlands 